\title{
Infinite-horizon optimal advertising in a market for durable goods
}

\author{
Thomas A. Weber ${ }^{*, \dagger}$ \\ Department of Management Science and Engineering, Stanford University, CA, U.S.A.
}

\begin{abstract}
SUMMARY
In this paper we analyse the optimal infinite-horizon advertising policy of a monopolist firm in a market for durable goods, based on classic models by Vidale-Wolfe (Oper. Res. 1957; 5(3):370-381) and NerloveArrow (Economica 1962; 29(114):129-142). A set of necessary conditions for optimality generalizing previous results is provided for the resulting non-convex system. In addition, we establish local (and in some cases global) asymptotic convergence of an optimal trajectory towards the unique optimal steady state. Copyright (C) 2005 John Wiley \& Sons, Ltd.
\end{abstract}

KEY WORDS: asymptotic stability; infinite-horizon optimal control; maximum principle

\section{INTRODUCTION}

There is a substantial literature on optimal advertising tackling the problem of finding the best advertising spending policy, possibly accompanied by other marketing mix variables such as price. Sethi [1] and Feichtinger et al. [2] provide comprehensive reviews, and it turns out that virtually all previous work considers the problem over a finite planning horizon $T$ with virtually no indication of how the length of $T$ should be determined. ${ }^{1}$ In that case the resulting optimal policy as well as the final state $x_{*}(T)$ may vary substantially with $T$, depending especially on the controllability properties of the system.

\footnotetext{
*Correspondence to: Thomas A. Weber, Department of Management Science and Engineering, Stanford University, Stanford, CA 94305-4026, U.S.A.

†E-mail: webert@stanford.edu

${ }^{1}$ Discussions about how the planning horizon should be chosen under uncertainty as a result of the tradeoff between improved operational decisions and cost for extending the horizon (e.g. due to the cost of collecting and processing additional information) can be found in the operations management literature; see, e.g. Reference [3].

Contract/grant sponsor: Stanford University

Contract/grant sponsor: University of Pennsylvania

Contract/grant sponsor: International Institute for Applied Systems Analysis
}

Copyright (C) 2005 John Wiley \& Sons, Ltd.

Received 21 June 2004

Revised 21 June 2005

Accepted 27 June 2005 
A central result of economic growth theory is the 'turnpike property' of capital accumulation, essentially stating that for sufficiently large $T$ optimal ('balanced' [4]) growth is achieved by steering the system from the initial state to an optimal steady state (or 'turnpike'), and then leaving the turnpike towards the end of the horizon to achieve short-term unsustainable superior growth. The turnpike property for finite-horizon optimal programs was first recognized by Dorfman et al. [4, p. 331], formalized in discrete-time by McKenzie [5, 6], and in continuous time among others by Cass [7], Cass and Shell [8], and Haurie [9] (see also Reference [10, Chapters 3 and 4]). For infinite-horizon problems a natural consequence of the turnpike property is global asymptotic convergence of the optimal trajectory, and such results have been provided under strong convexity assumptions on the (maximized) Hamiltonian by Cass and Shell [8], Brock and Scheinkman [11], and others. The convexity assumptions made there do not typically hold in models for optimal advertising or product diffusion, as saturation effects induced by a finite customer base may introduce non-convexities. Extensions of the results on global asymptotic convergence for a class of non-convex infinite-horizon optimal control problems have been provided by Feinstein and Luenberger [12] (who connect their algorithm for determining an optimal steady state to an earlier finding by Rockafellar [13]), or more generally by Carlson and Haurie [10, Chapter 6]. The latter results rest on the convexity-concavity of the Hamiltonian in a neighbourhood of an optimal steady state and/or the existence of trajectories supported by a convex set of system 'velocities'. Strong assumptions on the behaviour of the system outside a neighbourhood of the optimal steady state are needed to guarantee global asymptotic convergence of optimal trajectories. Since these restrictions are not easily satisfied for the optimal advertising model investigated here, we have adapted a local convergence result by Hartman [14] to fit the situation.

As a consequence of these results, it is natural to consider the problem of finding an optimal advertising policy over an infinite horizon, as this avoids unsustainable endpoint effects (for longer horizons) and variations of the optimal trajectory (for shorter horizons). Moreover, a 'going concern' for established brands and products is consistent with an infinite-horizon formulation of the problem. As a byproduct, one obtains an optimal steady state (i.e. the turnpike) that, in a sense, converts the abstract problem of profit maximization for the decision maker into a problem of most efficiently steering the system to this observable equilibrium point. ${ }^{2}$ This steady state can very often be expressed in metrics (e.g. advertising goodwill and installed base), for which estimation and measurement procedures can be found in the marketing literature $[15,16]$.

From a technical point of view, infinite-horizon optimal control problems typically have an incomplete set of necessary conditions that do not allow to select good candidates for an optimal solution effectively. Even though the Maximum Principle by Pontryagin et al. [17] has been extended to infinite-horizon problems by Arrow and Kurz [18] and Halkin [19], transversality conditions (i.e. additional conditions on the adjoint variables) are generally not available. ${ }^{3}$ In fact, Reference [19] provides a counterexample for a 'natural' extension of the finite-horizon transversality conditions, proposed by Arrow and Kurz [18]. ${ }^{4}$ Thus, some care is

\footnotetext{
${ }^{2}$ The optimal steady state is in most cases unique.

${ }^{3}$ Note that a slight modification of the proof of the classical Maximum Principle in Reference [17] is sufficient for a translation to the infinite-horizon optimal control problem. This has been already noted in Reference [17, pp. 189-191] (for the special case of a fixed terminal state).

${ }^{4}$ Arrow and Kurz were aware of this shortcoming (see footnote 1 on p. 46 in Reference [18]).
} 
necessary when formulating strong necessary conditions for infinite-horizon optimal control problems. Using the method of smooth approximation (reviewed by Aseev [20]), it is possible to obtain weak transversality conditions in the form of asymptotic stationarity of the (maximized) Hamiltonian and positivity of the adjoint variables (see References [21-23]). Here, instead of imposing growth limitations and monotonicity on state trajectories, we modify the results in References [21-23] to suit our situation, where the state space is a compact invariant set, which in turn allows us to drop some restrictive assumptions on the evolution of the states. Under these natural conditions for our problem, we are able to obtain exponential bounds on the adjoint variables (i.e. growth conditions) that are stronger than the Arrow-Kurz 'natural' transversality conditions mentioned earlier.

To illustrate our methods and the qualitative nature of the solutions, we have preferred a concrete problem parametrization rather than a perhaps less intuitive (and less conclusive) treatment of the problem in its full generality. In addition, we assume - in contrast to Reference [24] - decreasing returns to scale in advertising to concavify the Hamilton-Pontryagin function with respect to the control variable, leading to a unique and in most cases interior optimum in the class of admissible controls. This also avoids an often unrealistic 'bang-bang' intervention of the decision maker.

Outline: The next section will state the problem, report on the existence of an optimal solution based on Reference [25], and provide a simplifying equivalent reformulation. In Section 3, on the basis of the finite-horizon Pontryagin Maximum Principle [17], we will construct first-order necessary optimality conditions for our problem $(P)$ that include growth conditions in the form of upper and lower exponential bounds on the adjoint variables which converge to zero as time tends towards infinity. In Section 4 we will then qualitatively discuss optimal solutions to $(P)$ and their local asymptotic behaviour as a function of initial conditions and parameters. Section 5 concludes.

\section{STATEMENT OF THE PROBLEM}

Let us consider the following simple non-linear model for the accumulation of advertising effect $(y)$ in the Nerlove-Arrow form [24], and the evolution of the installed base $(z)$ in a form suggested by Vidale and Wolfe [26], normalized to the interval $[0,1]$. We include the effect of replacement sales due to obsolescence after a characteristic product lifetime of $1 / \beta$. The goal is to choose advertising effort $(u)$ such that total infinite-horizon discounted profits $\Pi$ are maximized. The evolution of the advertising effect (goodwill) and the installed base are then described by the following system of ordinary differential equations:

$$
\begin{gathered}
\dot{y}=u^{\kappa}-\alpha y \\
\dot{z}=(1-z) y-\beta z
\end{gathered}
$$

where $\alpha, \beta$ are positive constants, and the parameter $\kappa \in(0,1)$ describes the effect of decreasing returns to advertising effort. Equation (1), corresponding to the Nerlove-Arrow portion of the model, expresses the fact that in the absence of any advertising effort (i.e. when $u=0$ ) advertising goodwill decays exponentially with a characteristic time of $1 / \alpha$. More generally, advertising goodwill follows any step change in advertising effort exponentially with the same characteristic time of $1 / \alpha$. Equation (2), corresponding to the Vidale-Wolfe portion of the model, states that in each time unit the change in the installed base depends positively on the sale of $(1-z) y$ new products and the obsolescence of $\beta z$ old products. In equilibrium, the demand for new products 
$D(y, z)=(1-z) y$ equals supply. Sales are therefore proportional to the marketing effect $y$ and the fraction $1-z$ of consumers who currently do not own the product (either because they have not yet bought a unit or because their old product has become obsolete). ${ }^{5}$ If the market price (see footnote 7) for the product is $p$ (assumed fixed, for simplicity) and the marginal cost of advertising effort is equal to $c$, then the firm's aim is to maximize the discounted sum $\Pi$ of the firm's current-time profit $p D(y, z)-c u$. If we let $x=(y, z)^{\prime}$ be the state of the system, we can formulate the firm's dynamic profit-maximization problem in an optimal-control framework as follows:

$$
\Pi(x, u)=\int_{0}^{\infty} \mathrm{e}^{-\rho t}(p(1-z) y-c u) \mathrm{d} t \rightarrow \max
$$

subject to (1)-(2),

$$
x(0)=x_{0} \in \mathbb{R}_{+} \times[0,1]=: X
$$

and

$$
u \in[0, \bar{u}]=: U
$$

where $c, p, \rho, \bar{u}$ are appropriate positive constants, and $x_{0}=\left(y_{0}, z_{0}\right)^{\prime}$. Note that for any given initial condition (4) there is a compact invariant set $Y=[0,1+\bar{y}] \times[0,1] \subset X$, where $\bar{y}:=\bar{u}^{\kappa} / \alpha$. In particular, there is a time $\bar{T}\left(y_{0}\right)$, so that no matter what (admissible) control variable $u \in U$ is chosen, the system trajectory $x(t) \in Y$ for all $t \geqslant \bar{T}^{6}$. The constant $\rho$ denotes the discount rate, while $p$ represents the prevailing price in a market for durable goods, in which the firm is assumed to be a price taker. ${ }^{7}$ We will look for solutions $u$ in the space of bounded measurable functions. Any such function $u$ that satisfies the inclusion $u(t) \in U$ for all times $t$ is an admissible control. An admissible pair $(x, u)$ is any admissible control $u$ together with the corresponding trajectory $x$.

\subsection{Reformulation of the problem}

To simplify the resulting necessary optimality conditions, we rewrite the cost functional using $(2)^{8}$

$$
\Pi(x, u)=\int_{0}^{\infty} \mathrm{e}^{-\rho t}(p \dot{z}-\rho p z+(\beta+\rho p) z-c u) \mathrm{d} t=-p z_{0}+c \int_{0}^{\infty} \mathrm{e}^{-\rho t}(\gamma z-u) \mathrm{d} t
$$

where $\gamma:=(\beta+\rho p) / c$. Thus, we obtain the following equivalent formulation $(P)$ of the firm's infinite-horizon optimal control problem:

$$
(P): \quad J(x, u)=\int_{0}^{\infty} \mathrm{e}^{-\rho t}(\gamma z-u) \mathrm{d} t \rightarrow \max
$$

subject to (1)-(2) and (4)-(5). Economically speaking, the reformulation states that instead of maximizing discounted profits directly, it is possible to focus just on maximizing the discounted

\footnotetext{
${ }_{5}^{5}$ Note that consumers are assumed to be 'without memory' in the sense that after their old copy of the product becomes obsolete they need to be persuaded to buy a new copy of the product just as if they had never owned that product. This justifies the firm's ongoing interest in advertising and may be interpreted as a natural result of overlapping successive product generations in the absence of reputation effects other than those induced by advertising goodwill.

${ }^{6}$ It is clear that $\bar{T}=0$ for $y_{0} \leqslant 1+\bar{y}$; for $y_{0}>1+\bar{y}$ one can use $\bar{T}\left(y_{0}\right)=\frac{1}{\alpha} \ln \left(y_{0}-\bar{y}-1\right)$.

${ }^{7}$ More precisely, at non-zero unit costs $p$ is the constant absolute profit margin per unit sold. For simplicity one may think of $p$ as price in the case of zero unit cost.

${ }^{8}$ Here we use the fact that $z$ is uniformly bounded, as can be easily seen from (2); in fact, $z \in[0,1]$.
} 
sum of the difference between weighted installed base and advertising expenditures. The constant positive weight $\gamma$ of the installed base versus advertising expenditures (measured, for instance, in amount per time unit) increases with price (or markup) $p$, discard rate $\beta$, and discount rate $\rho$.

\subsection{Existence of an optimal solution}

As pointed out before, the state space $X$ contains a compact invariant subset $Y$ that is reached in finite time, and thus all admissible trajectories are uniformly bounded. In addition, the current value of the integrand of the objective functional $J$

$$
h(z, u):=\gamma z-u
$$

is bounded, since for any $(z, u) \in[0,1] \times U$

$$
-\bar{u} \leqslant h(z, u) \leqslant \gamma
$$

Thus, there is a non-increasing positive function $\omega: \mathbb{R}_{+} \rightarrow \mathbb{R}$ such that

$$
\lim _{t \rightarrow \infty} \omega(t)=0
$$

and, for any admissible pair $(x, u)$ of system (1)-(2), subject to (4)-(5), we have

$$
\int_{T}^{\infty} \mathrm{e}^{-\rho t}|h(z, u)| \mathrm{d} t \leqslant \omega(T)
$$

for all $T>0$. In view of (7), we can put without loss of generality

$$
\omega(T)=\frac{m \mathrm{e}^{-\rho T}}{\rho}
$$

where $m:=\max \{\bar{u}, \gamma\}$. With this, Theorem 3.6 in Reference [25] guarantees the existence of a solution to the infinite-horizon optimal control problem $(P){ }^{9}$

\section{SMOOTH APPROXIMATION OF NECESSARY OPTIMALITY CONDITIONS}

In this section we follow the general approach by Aseev et al. [23] and construct necessary optimality conditions for our problem $(P)$ by considering a sequence of classical optimal control problems $\left(P_{k}\right)$ where each is each defined on its own finite time interval $\left[0, T_{k}\right]$, where $0<T_{k} \leqslant T_{k+1}$, and $T_{k} \rightarrow \infty$ as $k \rightarrow \infty$. Our problem $(P)$ does not satisfy the assumptions in Reference [23] directly, so that a number of modifications need to be made. The resulting maximum principle contains growth conditions in the form of exponential bounds on the adjoint variables that are stronger than the asymptotic stationarity of the Hamiltonian as obtained in Reference [23], and stronger than the 'natural' transversality conditions by Arrow and Kurz [18], $\lim _{t \rightarrow \infty} \psi(t)=0$. We also characterize the latter in terms of the initial values $\psi(0)$.

\footnotetext{
$\overline{{ }^{9} \text { An appropriate, }}$ simple and direct existence proof can also be found in Reference [21]. 


\subsection{Auxiliary constructions}

Assume that $\left(x_{*}, u_{*}\right)$ is an optimal pair for the original infinite-horizon optimal control problem $(P)$. Take a sequence of real-valued functions $v_{k} \in C^{1}\left(\mathbb{R}_{+}\right)$, and positive constants $\sigma_{k}<\infty$ (with $\left.\sigma_{k} \leqslant \sigma_{k+1}\right), k=1,2, \ldots$, such that

$$
\begin{gathered}
\left\|v_{k}\right\|_{\infty} \leqslant 1+\bar{u} \\
\int_{0}^{\infty} \mathrm{e}^{-\rho t}\left(v_{k}-u_{*}\right)^{2} \mathrm{~d} t \leqslant \frac{1}{k} \\
\left\|\dot{v}_{k}\right\|_{\infty} \leqslant \sigma_{k}
\end{gathered}
$$

and

$$
\begin{gathered}
\omega\left(T_{k}\right) \leqslant \frac{1}{k\left(1+\sigma_{k}\right)} \\
\lim _{k \rightarrow \infty} \sigma_{k}=\infty
\end{gathered}
$$

Such a sequence $\left\{v_{k}, \sigma_{k}\right\}$ exists and can be obtained using standard approximation methods. Let us now consider a sequence of the following classical optimal control problems $\left(P_{k}\right)$ :

$$
\left(P_{k}\right): \quad J_{k}(x, u)=\int_{0}^{T_{k}} \mathrm{e}^{-\rho t}\left(h(z, u)-\frac{\left(v_{k}-u\right)^{2}}{1+\sigma_{k}}\right) \mathrm{d} t \rightarrow \max
$$

subject to (1)-(2) and (4)-(5), $k=1,2 \ldots$ By the Filippov Existence Theorem [27, p. 314], there exists an optimal control $u_{k}$ solving $\left(P_{k}\right)$, and we assume that $u_{k}$ and its associated trajectory $x_{k}=\left(y_{k}, z_{k}\right)^{\prime}$ are extended in an arbitrary admissible way onto $\mathbb{R}_{+}$, so that $\left(x_{k}, u_{k}\right)$ forms an admissible pair for $(P)$.

Proposition 1

Let $T>0$. Then

$$
u_{k} \rightarrow u_{*} \quad \text { in } L_{2}[0, T] \text { as } k \rightarrow \infty
$$

\section{Proof}

Fix $\varepsilon, T>0$ and an integer $k_{0}$ such that $T \geqslant T_{k_{0}}$. Then we have for $k \geqslant k_{0}$

$$
\begin{aligned}
J_{k}\left(x_{k}, u_{k}\right) & =\int_{0}^{T_{k}} \mathrm{e}^{-\rho t}\left(h\left(z_{k}, u_{k}\right)-\frac{\left(v_{k}-u_{k}\right)^{2}}{1+\sigma_{k}}\right) \mathrm{d} t \\
& \leqslant \int_{0}^{T_{k}} \mathrm{e}^{-\rho t} h\left(z_{k}, u_{k}\right) \mathrm{d} t-\frac{\mathrm{e}^{-\rho T}}{1+\sigma_{k}} \int_{0}^{T}\left(v_{k}-u_{k}\right)^{2} \mathrm{~d} t
\end{aligned}
$$


Hence, by optimality of $\left(x_{k}, u_{k}\right)$ for $\left(P_{k}\right)$ and by optimality of $\left(x_{*}, u_{*}\right)$ for $(P)$ as well as using (8) and (10)

$$
\begin{aligned}
\frac{\mathrm{e}^{-\rho T}}{1+\sigma_{k}} \int_{0}^{T}\left(v_{k}-u_{k}\right)^{2} \mathrm{~d} t & \leqslant \int_{0}^{T_{k}} \mathrm{e}^{-\rho t} h\left(z_{k}, u_{k}\right) \mathrm{d} t-J_{k}\left(x_{*}, u_{*}\right) \\
& \leqslant \int_{0}^{T_{k}} \mathrm{e}^{-\rho t} h\left(z_{k}, u_{k}\right) \mathrm{d} t-\left(J\left(x_{*}, u_{*}\right)-\omega\left(T_{k}\right)-\int_{0}^{\infty} \mathrm{e}^{-\rho t} \frac{\left(v_{k}-u_{*}\right)^{2}}{1+\sigma_{k}} \mathrm{~d} t\right) \\
& \leqslant J\left(x_{k}, u_{k}\right)+\omega\left(T_{k}\right)-\left(J\left(x_{*}, u_{*}\right)-\omega\left(T_{k}\right)-\frac{1}{k\left(1+\sigma_{k}\right)}\right) \\
& \leqslant J\left(x_{k}, u_{k}\right)-J\left(x_{*}, u_{*}\right)+\frac{3}{k\left(1+\sigma_{k}\right)} \\
& \leqslant \frac{3}{k\left(1+\sigma_{k}\right)}
\end{aligned}
$$

Therefore, for $k \geqslant \max \left\{k_{0}, 16 \mathrm{e}^{\rho T} / \varepsilon^{2}\right\}$ we have using (10)

$$
\left\|u_{k}-u_{*}\right\|_{L_{2}[0, T]} \leqslant\left\|u_{k}-v_{k}\right\|_{L_{2}[0, T]}+\left\|v_{k}-u_{*}\right\|_{L_{2}[0, T]} \leqslant \frac{(1+3) \sqrt{\mathrm{e}^{\rho T}}}{\sqrt{k}} \leqslant \varepsilon
$$

which concludes the proof.

\section{Remark}

Proposition 1 guarantees that for any $T>0$, as $k \rightarrow \infty$

$$
\begin{gathered}
u_{k} \rightarrow u_{*} \quad \text { in } L_{2}[0, T] \\
x_{k} \rightrightarrows x_{*} \quad \text { on }[0, T] \\
\dot{x}_{k} \rightarrow \dot{x}_{*} \quad \text { weakly in } L_{1}[0, T]
\end{gathered}
$$

Without loss of generality (by selecting a subsequence if necessary) we can assume that $u_{k}(t) \stackrel{\text { a.e. }}{\longrightarrow} u_{*}(t)$. The strong convergence in (16) is then a consequence of (15), the absolute continuity of $x$ and the boundedness of system equations (1)-(2) (see e.g. Reference [28, Theorem 7, p. 12]). The weak convergence in (17) is due to the Dunford-Pettis property of $L_{1}$ [29, IV.8.9, p. 292].

\subsection{Necessary optimality conditions for $(P)$}

We will now formulate a set of necessary conditions that generalize the Maximum Principle proved by Pontryagin et al. [17, pp. 189-191]. Our approach here, as in the last subsection, closely follows the approach in Reference [23]. First, consider the Hamilton-Pontryagin function

$$
\mathscr{H}\left(t, x, u, \psi^{0}, \psi\right)=\psi^{0} \mathrm{e}^{-\rho t}(\gamma z-u)+\lambda\left(u^{\kappa}-\alpha y\right)+\mu((1-z) y-\beta z)
$$


where $\psi^{0} \geqslant 0$ is a constant, and $\psi(t)=(\lambda(t), \mu(t))^{\prime}$ are the adjoint variables for this problem. Whenever $\psi^{0}>0$ it is possible, without loss of generality, to take $\psi^{0}=1$, just by renormalizing the adjoint variables $\lambda, \mu$. This simplification (to normal form) will be rigorously justified in our proof of the necessary optimality conditions below. ${ }^{10}$ Everywhere below it is assumed that $\psi_{0}=1$. The (maximized) Hamiltonian function is defined as

$$
H(t, x, \psi)=\sup _{u \in U} \mathscr{H}(t, x, u, \psi)
$$

and straightforward maximization of $\mathscr{H}$ gives

$$
u_{*}= \begin{cases}\min \left\{\bar{u},\left(\kappa \lambda \mathrm{e}^{\rho t}\right)^{1 / 1-\kappa}\right\}, & \lambda \geqslant 0 \\ 0, & \lambda<0\end{cases}
$$

The next proposition provides necessary optimality conditions for our problem $(P)$, including a growth condition that implies the transversality condition and asymptotic stationarity of the Hamiltonian obtained in Reference [23].

Proposition 2 (Necessary optimality conditions)

Let $u_{*}$ be an optimal solution of $(P)$, and $x_{*}=\left(y_{*}, z_{*}\right)^{\prime}$ the corresponding trajectory. Then there exists an absolutely continuous function $\psi: \mathbb{R}_{+} \rightarrow \mathbb{R}^{2}$ such that the following conditions are satisfied:

(i) The function $\psi=(\lambda, \mu)^{\prime}$ is a solution to the adjoint system $\dot{\psi}=-\frac{\partial \mathscr{H}\left(t, u_{*}, x_{*}, \psi\right)}{\partial x}$, i.e.

$$
\begin{gathered}
\dot{\lambda}=\alpha \lambda-\mu\left(1-z_{*}\right) \\
\dot{\mu}=-\gamma \mathrm{e}^{-\rho t}+\mu\left(\beta+y_{*}\right)
\end{gathered}
$$

(ii) The maximality condition

$$
\mathscr{H}\left(t, x_{*}(t), u_{*}(t), \psi(t)\right) \stackrel{\text { a.e. }}{=} H\left(t, x_{*}(t), \psi(t)\right)
$$

holds a.e. on $\mathbb{R}_{+}$.

(iii) The functions $\lambda(t), \mu(t)$ are strictly positive on $\mathbb{R}_{+}$. Moreover, the following growth conditions are satisfied for all $t \in \mathbb{R}_{+}$

$$
\frac{\gamma\left(1-\max \left\{z_{0}, \bar{z}\right\}\right) \mathrm{e}^{-\rho t}}{(\rho+\alpha)\left(\rho+\beta+\max \left\{y_{0}, \bar{y}\right\}\right)} \leqslant \lambda(t) \leqslant \frac{\gamma \mathrm{e}^{-\rho t}}{(\rho+\alpha)(\rho+\beta)}
$$

and

$$
\frac{\gamma \mathrm{e}^{-\rho t}}{\rho+\beta+\max \left\{y_{0}, \bar{y}\right\}} \leqslant \mu(t) \leqslant \frac{\gamma \mathrm{e}^{-\rho t}}{\rho+\beta}
$$

where $\bar{z}:=\bar{y} /(\beta+\bar{y})$.

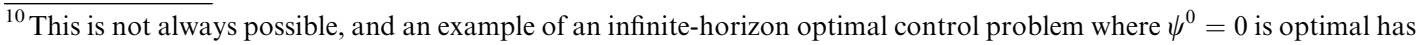
been provided by Halkin [19].
} 


\section{Remark}

(a) The growth conditions (23)-(24) can be written in the following more compact form:

$$
\lambda(t) \in[\underline{\lambda}, \bar{\lambda}] \mathrm{e}^{-\rho t}, \quad \mu(t) \in[\underline{\mu}, \bar{\mu}] \mathrm{e}^{-\rho t} \text { for all } t \in \mathbb{R}_{+}
$$

with obvious definitions of the constants $\underline{\lambda}, \bar{\lambda}, \underline{\mu}, \bar{\mu}$. Clearly these growth conditions imply the transversality conditions in Arrow-Kurz form.

(b) Relation (19) together with part (iii) of Proposition 2 can be used to rewrite the state equations (1)-(2) to

$$
\begin{gathered}
\dot{y}=\left(\kappa \lambda \mathrm{e}^{\rho t}\right)^{\kappa / 1-\kappa}-\alpha y \\
\dot{z}=(1-z) y-\beta z
\end{gathered}
$$

as long as

$$
\bar{u} \geqslant(\kappa \bar{\lambda})^{1 / 1-\kappa}
$$

If on the other hand $\bar{u} \leqslant(\kappa \underline{\lambda})^{1 / 1-\kappa}$, then the optimal control is constant, $u_{*}=\bar{u}$, and the corresponding optimal state trajectory can be given explicitly. ${ }^{11}$ In the intermediate case where $\bar{u}^{1-\kappa} / \kappa \in(\underline{\lambda}, \bar{\lambda})$, the right-hand side of system equation (1) can in principle exhibit non-smooth behaviour, as the optimal control may intermittently go into saturation.

(c) The (maximized) Hamiltonian is not concave in $x$ (and not convex). For instance, the second derivative of $H$ with respect to $y$ vanishes, and thus the two eigenvalues of the Hessian of $H$ cannot have the same (negative) sign. More precisely, from (18) using (19) we have that ${ }^{12}$

$$
H(t, x, \psi)=-\mu z y+\ell(t, x, \psi)
$$

where $\ell: \mathbb{R}_{+} \times X \times \mathbb{R}^{2} \rightarrow \mathbb{R}$ is a smooth function, affine in $x=(y, z)^{\prime}$. Clearly, along the line $z=y$ the Hamiltonian $H$ is concave, while along the line $y=1-z$ it is convex, since then $H=\mu z^{2}-\mu z+\ell$.

\section{Proof}

Considering the sequence of classical optimal control problems $\left(P_{k}\right)$ constructed above, let $u_{k}$ be an optimal solution of $\left(P_{k}\right)$ and $x_{k}=\left(y_{k}, z_{k}\right)^{\prime}$ the corresponding trajectory for $k=1,2, \ldots$ By the Pontryagin Maximum Principle [17] there exists an absolutely continuous function $\psi_{k}=$ $\left(\lambda_{k}, \mu_{k}\right)^{\prime}: \mathbb{R}_{+} \rightarrow \mathbb{R}^{2}$ such that the following necessary optimality conditions for $\left(P_{k}\right)$ hold:

$$
\begin{gathered}
\dot{\lambda}_{k}=\alpha \lambda_{k}-\mu_{k}\left(1-z_{k}\right) \\
\dot{\mu}_{k}=-\gamma \mathrm{e}^{-\rho t}+\mu_{k}\left(\beta+y_{k}\right)
\end{gathered}
$$

\footnotetext{
$\overline{{ }^{11} \mathrm{~A} \text { discussion of }}$ trajectories under constant controls can be found in Section 6.1.

${ }^{12}$ By part (iv) of Proposition 2 the adjoint variables $\lambda, \mu$ are positive.
} 


$$
\begin{gathered}
\mathscr{H}_{k}\left(t, x_{k}(t), u_{k}(t), \psi_{k}(t)\right) \stackrel{\text { a.e. }}{=} H_{k}\left(t, x_{k}(t), \psi_{k}(t)\right) \\
\psi_{k}\left(T_{k}\right)=0
\end{gathered}
$$

where we have used the expression $\mathscr{H}_{k}$ for the Hamilton-Pontryagin function in normal form ${ }^{13}$

$$
\mathscr{H}_{k}\left(t, x_{k}(t), u_{k}(t), \psi_{k}(t)\right)=\mathrm{e}^{-\rho t} h\left(z_{k}, u_{k}\right)+\lambda\left(u_{k}^{\kappa}-\alpha y_{k}\right)+\mu\left(\left(1-z_{k}\right) y_{k}-\beta z_{k}\right)
$$

and $H_{k}$ for the corresponding (maximized) Hamiltonian

$$
H_{k}\left(t, x_{k}, \psi_{k}\right)=\sup _{u \in U} \mathscr{H}_{k}\left(t, x_{k}, u, \psi_{k}\right)
$$

with respect to problem $\left(P_{k}\right)$. We will now concentrate on proving part (iii) of the proposition. From the boundary condition (32) and adjoint equation (30) we have by the variation-ofconstants formula [30, pp. 75-76]

$$
\mu_{k}(t)=\gamma \int_{t}^{T_{k}} \exp \left(-\rho \theta-\int_{t}^{\theta}\left(\beta+y_{k}(s)\right) \mathrm{d} s\right) \mathrm{d} \theta>0
$$

for all $t \in\left[0, T_{k}\right)$. Therefore

$$
\mu_{k}(t) \leqslant \gamma \int_{t}^{T_{k}} \exp (-\rho \theta-\beta(\theta-t)) \mathrm{d} \theta=\frac{\gamma}{\rho+\beta}\left(\mathrm{e}^{-\rho t}-\mathrm{e}^{-\rho T_{k}} \mathrm{e}^{-\beta\left(T_{k}-t\right)}\right) \leqslant \frac{\gamma \mathrm{e}^{-\rho t}}{\rho+\beta}
$$

and also

$$
\mu_{k}(t) \geqslant \gamma \int_{t}^{T_{k}} \exp \left(-\rho \theta-\left(\beta+m_{y}\right)(\theta-t)\right) \mathrm{d} \theta=\frac{\gamma\left(\mathrm{e}^{-\rho t}-\mathrm{e}^{-\rho T_{k}} \mathrm{e}^{-\left(\beta+m_{y}\right)\left(T_{k}-t\right)}\right)}{\rho+\beta+m_{y}}
$$

for all $t \in\left[0, T_{k}\right.$ ), where we have set $m_{y}:=\max \left\{y_{0}, \bar{y}\right\}$. Similarly, we obtain from (30), (32), and (33)

$$
\lambda_{k}(t)=\mathrm{e}^{\alpha t} \int_{t}^{T_{k}} \mathrm{e}^{-\alpha \theta} \mu_{k}(\theta)\left(1-z_{k}(\theta)\right) \mathrm{d} \theta>0
$$

for all $t \in\left[0, T_{k}\right)$, since $z_{k}(t)<1$ on $\left(0, T_{k}\right)$. Using the above bounds on $\mu_{k}$, given by (34)-(35), this implies

$$
\lambda_{k}(t) \leqslant \mathrm{e}^{\alpha t} \int_{t}^{T_{k}} \mathrm{e}^{-\alpha \theta} \mu_{k}(\theta) \mathrm{d} \theta \leqslant \frac{\gamma\left(\mathrm{e}^{-\rho t}-\mathrm{e}^{-\rho T_{k}-\alpha\left(T_{k}-t\right)}\right)}{(\rho+\alpha)(\rho+\beta)} \leqslant \frac{\gamma \mathrm{e}^{-\rho t}}{(\rho+\alpha)(\rho+\beta)}
$$

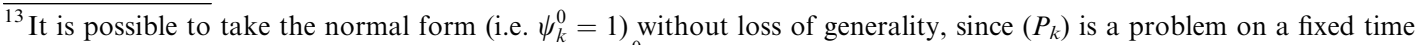
interval $\left[0, T_{k}\right]$ with free terminal state, and thus $\psi_{k}^{0} \neq 0$. 
and if we use the abbreviation $m_{z}:=\max \left\{z_{0}, \bar{z}\right\}$

$$
\begin{aligned}
\lambda_{k}(t) & \geqslant \frac{\gamma\left(1-m_{z}\right) \mathrm{e}^{\alpha t}}{\rho+\beta+m_{y}} \int_{t}^{T_{k}} \mathrm{e}^{-\alpha \theta}\left(\mathrm{e}^{-\rho \theta}-\mathrm{e}^{-\rho T_{k}} \mathrm{e}^{-\left(\beta+m_{y}\right)\left(T_{k}-\theta\right)}\right) \mathrm{d} \theta \\
& \geqslant \frac{\gamma\left(1-m_{z}\right) \mathrm{e}^{\alpha t}}{\rho+\beta+m_{y}}\left(\frac{\mathrm{e}^{-(\rho+\alpha) t}-\mathrm{e}^{-(\rho+\alpha) T_{k}}}{\alpha+\rho}-\frac{\mathrm{e}^{-\rho T_{k}}}{\rho+\alpha+m_{y}}\left(\mathrm{e}^{-\alpha t}-\mathrm{e}^{-\alpha T_{k}} \mathrm{e}^{-\left(\beta+m_{y}\right)\left(T_{k}-t\right)}\right)\right) \\
& =\frac{\gamma\left(1-m_{z}\right)}{\rho+\beta+m_{y}}\left(\frac{\mathrm{e}^{-\rho t}-\mathrm{e}^{-\alpha T_{k}} \mathrm{e}^{-\rho\left(T_{k}-t\right)}}{\rho+\alpha}-\frac{\mathrm{e}^{-\rho T_{k}}\left(1-\mathrm{e}^{-\left(\alpha+\beta+m_{y}\right)\left(T_{k}-t\right)}\right)}{\rho+\alpha+m_{y}}\right)
\end{aligned}
$$

for all $t \in\left[0, T_{k}\right]{ }^{14}$

Consider now the increasing sequence of time intervals $I_{j}=\left[0, T_{j}\right], j=1,2, \ldots$, and on each $I_{j}$ consider the sequences $\left\{u_{k}\right\},\left\{x_{k}\right\}$, and $\left\{\psi_{k}\right\}$. Given $I_{j}$, the sequence $\left\{\left\|\psi_{k}(0)\right\|\right\}$ is uniformly bounded. Using the Bellman-Gronwall Lemma [31, pp. 474-475] and the adjoint equations (29)-(30) one can assume without loss of generality that there exists an absolutely continuous function $\psi=\left(\lambda_{k}, \mu_{k}\right)^{\prime}: \mathbb{R}_{+} \rightarrow \mathbb{R}^{2}$ such that (as $\left.k \rightarrow \infty\right)$

$$
\psi_{k} \rightrightarrows \psi \quad \text { on } I_{j}
$$

and

$$
\dot{\psi}_{k} \rightarrow \dot{\psi} \quad \text { weakly in } L_{1}\left(I_{j}\right)
$$

Given the sequence $\left\{I_{j}\right\}$ we can pass on each $I_{j}, j=1,2, \ldots$, from the sequence $\left\{\psi_{k}\right\}$ to a subsequence converging to $\psi$. By selecting a diagonal subsequence, one can assume that there is an absolutely continuous function $\psi: \mathbb{R}_{+} \rightarrow \mathbb{R}$ such that, for any $T>0$, we have $($ as $k \rightarrow \infty)$

$$
\begin{gathered}
\psi_{k} \rightrightarrows \psi \quad \text { on }[0, T] \\
\dot{\psi}_{k} \rightarrow \dot{\psi} \quad \text { weakly in } L_{1}[0, T]
\end{gathered}
$$

By Proposition 1 and the discussion thereafter, we have that $u_{k} \rightarrow u_{*}$ strongly in $L_{2}[0, T]$ as $k \rightarrow \infty$, and $x_{k} \rightrightarrows x_{*}$ uniformly on $[0, T]$ as $k \rightarrow \infty$. Using the Mazur theorem (see e.g. Reference [32]), the absolutely continuous function $\psi$ is a solution to the adjoint system (29)-(30) on any time interval $[0, T], T>0$. This proves part (i).

The maximality condition (22) follows from passing to the limit in (31). This proves part (ii).

The strict positivity of $\psi$ is a direct consequence ${ }^{15}$ of (35) and (38) for $k \rightarrow \infty$. Similarly, relations (23)-(24) are obtained from (34)-(35) and (37)-(38) by passing to the limit for $k \rightarrow \infty$. Thus we have shown part (iii), which concludes the proof.

\section{Remark}

(a) The state trajectory is bounded, i.e. $x_{*}(t) \in\left[0, \max \left\{y_{0}, \bar{y}\right\}\right] \times\left[0, \max \left\{z_{0}, \bar{z}\right\}\right]$ for all $t \in \mathbb{R}_{+}$. Thus the growth conditions (23)-(24), together with the boundedness of $h$, imply the

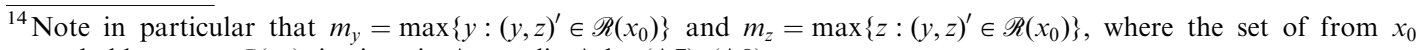
reachable states, $\mathscr{R}\left(x_{0}\right)$, is given in Appendix A by (A7)-(A8).

${ }^{15}$ In the special case where $z_{0}=1$, note that $m_{z}=1$, but also that nevertheless $z_{*}(t)<1$ for all $t>0$, since $\dot{z}<0$ for $z>\bar{z}$ by (2) and $\bar{z}<1$.
} 
asymptotic stationarity of the Hamiltonian,

$$
\lim _{t \rightarrow \infty} H\left(t, x_{*}(t), \psi(t)\right)=0
$$

(b) From (20)-(22) we get that

$$
\frac{\mathrm{d}}{\mathrm{d} t} H\left(t, x_{*}(t), \psi(t)\right) \stackrel{\text { a.e. }}{=} \frac{\partial \mathscr{H}}{\partial t}\left(t, x_{*}(t), u_{*}(t), \psi(t)\right)=-\rho \mathrm{e}^{-\rho t} h\left(z_{*}(t), u_{*}(t)\right)
$$

which, integrated over $\mathbb{R}_{+}$, together with (40) yields

$$
H\left(0, x_{0}, \psi(0)\right)=\rho \int_{0}^{\infty} \mathrm{e}^{-\rho t} h\left(z_{*}, u_{*}\right) \mathrm{d} t=\rho J\left(x_{*}, u_{*}\right)
$$

Thus, we have obtained an expression for $J_{*}:=J\left(x_{*}, u_{*}\right)$ in terms of the initial conditions $x(0)=\left(y_{0}, z_{0}\right)^{\prime}, \psi(0)=\left(\lambda_{0}, \mu_{0}\right)^{\prime}$

$$
\begin{aligned}
J_{*}= & \frac{1}{\rho} \max _{u \in U}\left\{h\left(z_{0}, u\right)+\lambda_{0}\left(u^{\kappa}-\alpha y_{0}\right)+\mu_{0}\left(\left(1-z_{0}\right) y_{0}-\beta z_{0}\right)\right\} \\
= & \frac{\gamma z_{0}}{\rho}+\frac{1}{\rho}\left(\lambda_{0} \min \left\{\bar{u}^{\kappa},\left(\kappa \lambda_{0}\right)^{\kappa / 1-\kappa}\right\}-\min \left\{\bar{u},\left(\kappa \lambda_{0}\right)^{1 / 1-\kappa}\right\}-\alpha \lambda_{0} y_{0}\right) \\
& +\frac{\mu_{0}}{\rho}\left(\left(1-z_{0}\right) y_{0}-\beta z_{0}\right)
\end{aligned}
$$

In the special case where (28) holds, we have

$$
J_{*}=\frac{\beta z_{0}+\left(\frac{1}{\kappa}-1\right)\left(\kappa \lambda_{0}\right)^{1 / 1-\kappa}-\alpha \lambda_{0} y_{0}}{\rho}+\frac{\mu_{0}}{\rho}\left(\left(1-z_{0}\right) y_{0}-\beta z_{0}\right) \text { for } \bar{u} \geqslant(\kappa \bar{\lambda})^{1 / 1-\kappa}
$$

Proposition 2 provides growth conditions in the form of inequalities, stronger than the 'natural' transversality condition ${ }^{16}$

$$
\lim _{t \rightarrow \infty} \psi(t)=0
$$

proposed for instance by Arrow and Kurz [18], which is a direct consequence of (23)-(24). We will now provide a characterization of the 'natural' transversality (42) in terms of initial values of the adjoint variable $\psi$.

\section{Proposition 3 ('Natural' transversality)}

Let $\left(x_{*}, u_{*}\right)$ be an optimal pair for $(P)$ and $\psi(t)=(\lambda(t), \mu(t))^{\prime}$ an adjoint variable satisfying the conditions in Proposition 2. The 'natural' transversality condition (42) holds if and only if the

\footnotetext{
${ }^{16}$ Such a transversality condition need not hold, even for simple infinite-horizon optimal control problems. An appropriate counterexample was given by Halkin [19].
} 
following two relations are true:

$$
\begin{gathered}
\lambda(0)=\lim _{t \rightarrow \infty} \int_{0}^{t} \mathrm{e}^{-\alpha \theta} \mu(\theta)\left(1-z_{*}(\theta)\right) \mathrm{d} \theta \\
\mu(0)=\gamma \lim _{t \rightarrow \infty} \int_{0}^{t} \exp \left(-(\rho+\beta) \theta-\int_{0}^{\theta} y_{*}(s) \mathrm{d} s\right) \mathrm{d} \theta
\end{gathered}
$$

\section{Proof}

' $\Rightarrow$ ': Given initial conditions $\lambda(0)$ and $\mu(0)$, the solutions of the system (20)-(21) have the form

$$
\begin{gathered}
\lambda(t)=\mathrm{e}^{\alpha t}\left(\lambda(0)-\int_{0}^{t} \mathrm{e}^{-\alpha \theta} \mu(\theta)\left(1-z_{*}(\theta)\right) \mathrm{d} \theta\right) \\
\mu(t)=\left[\mu(0)-\gamma \int_{0}^{t} \exp \left(-(\rho+\beta) \theta-\int_{0}^{\theta} y_{*}(s) \mathrm{d} s\right) \mathrm{d} \theta\right] \exp \left(\int_{0}^{t}\left(\beta+y_{*}(\theta)\right) \mathrm{d} \theta\right)
\end{gathered}
$$

Thus, relations (43)-(44) are an immediate consequence of (42).

' $\Leftarrow$ ': Starting from (46) let us first examine the limit of $\mu(t)$ as $t \rightarrow \infty$

$$
\lim _{t \rightarrow \infty} \mu(t)=\lim _{t \rightarrow \infty} \frac{\mu(0)-\gamma \int_{0}^{t} \exp \left(-(\rho+\beta) \theta-\int_{0}^{\theta} y_{*}(s) \mathrm{d} s\right) \mathrm{d} \theta}{\exp \left(-\int_{0}^{t}\left(\beta+y_{*}(\theta)\right) \mathrm{d} \theta\right)}
$$

Both the numerator and denominator of the right-hand side of the last expression tend towards zero as $t \rightarrow \infty$. Thus, by L'Hospital's rule we have

$$
\lim _{t \rightarrow \infty} \mu(t)=\lim _{t \rightarrow \infty} \frac{-\gamma \exp \left(-\rho t-\int_{0}^{t}\left(\beta+y_{*}(\theta)\right) \mathrm{d} \theta\right)}{-\left(\beta+y_{*}(t)\right) \exp \left(-\int_{0}^{t}\left(\beta+y_{*}(\theta)\right) \mathrm{d} \theta\right)}=\lim _{t \rightarrow \infty} \frac{\gamma \mathrm{e}^{-\rho t}}{\beta+y_{*}(t)}=0
$$

Based on (45), let us compute the limit of $\lambda(t)$ as $t \rightarrow \infty$

$$
\lim _{t \rightarrow \infty} \lambda(t)=\lim _{t \rightarrow \infty} \mathrm{e}^{\alpha t}\left(\lambda(0)-\int_{0}^{t} \mathrm{e}^{-\alpha \theta} \mu(\theta)\left(1-z_{*}(\theta)\right) \mathrm{d} \theta\right)=\lim _{t \rightarrow \infty} \frac{\lambda(0)-\int_{0}^{t} \mathrm{e}^{-\alpha \theta} \mu(\theta)\left(1-z_{*}(\theta)\right) \mathrm{d} \theta}{\mathrm{e}^{-\alpha t}}
$$

Again both the numerator and denominator of the last expression tend towards zero as $t \rightarrow \infty$, so that we obtain by L'Hospital's rule and (47)

$$
\lim _{t \rightarrow \infty} \lambda(t)=\lim _{t \rightarrow \infty} \frac{-\mathrm{e}^{-\alpha t} \mu(t)\left(1-z_{*}(t)\right)}{-\alpha \mathrm{e}^{-\alpha . t}}=\lim _{t \rightarrow \infty} \frac{\mu(t)}{\alpha}\left(1-z_{*}(t)\right)=0
$$

which concludes the proof.

\section{Remark}

(a) The second part of the proof (' $\Leftarrow$ ') indeed uses the transversality condition (42), as it is the prerequisite for the application of l'Hospital's rule. 
(b) The proof tacitly uses the fact that $X$ contains a bounded and invariant set $Y$ which is reached in finite time, so that $z_{*}(t)$ in particular is uniformly bounded (only the positivity of $y_{*}(t)$ matters in the proof).

\section{SOLUTION OF THE INFINITE-HORIZON OPTIMAL CONTROL PROBLEM}

The best equilibrium state $x_{\mathrm{b}}^{0}$ maximizes the integrand $h$ of the objective function $J$ for all times $t$, subject to leaving system (1)-(2) at rest. On the other hand, a system that starts at the best equilibrium state may increase the value of the objective function by leaving the best equilibrium state tending towards an optimal steady state that provides maximum growth of discounted profits, allowing for intertemporal increases in profits by passing through nonequilibrium states. For a zero discount rate $(\rho=0)$ both concepts coincide. In the following, we explicitly determine these states and provide sufficient conditions for the asymptotic convergence of an optimal trajectory towards the optimal steady state, provided one starts close enough to that state. In addition, we discuss the synthesis of an optimal, profit-maximizing advertising policy.

\subsection{Best equilibrium state $x_{\mathrm{b}}^{0}$}

Consider first the set of possible stationary states $S$, where necessarily $\dot{x}=0$. Using (1)-(2) and (5) we obtain

$$
S=\left\{(y, z) \in X: z=\frac{y}{\beta+y}, y \in[0, \bar{y}]\right\}
$$

Clearly we have that $S$ is a one-dimensional compact manifold, with $\partial S=\{0, \bar{x}\}$. We would like to determine a maximizer of the integrand of $J(x, u)$ in (3) on $S$, which we term best equilibrium state $x_{\mathrm{b}}^{0}$ (Figure 1). For this fix $t \geqslant 0$ and rewrite the integrand in (6) on $S$ subject to $\dot{x}=0$

$$
\left\{h(z, u):(y, z) \in S, u^{\kappa}=\alpha y\right\} \ni \frac{\gamma y}{\beta+y}-(\alpha y)^{1 / \kappa}=: h_{S}(y)
$$

where $h_{S}: \mathbb{R}_{+} \rightarrow \mathbb{R}$ is a smooth function. The necessary condition for an interior optimum can be written in the form

$$
h_{S}^{\prime}(y)=\frac{\gamma \beta}{(\beta+y)^{2}}-\frac{\alpha^{1 / \kappa}}{\kappa} y^{1-\kappa / \kappa}=0
$$

and together with

$$
h_{S}^{\prime \prime}(y)=-2\left(\frac{\gamma \beta}{(\beta+y)^{3}}+\frac{\alpha^{1 / \kappa}}{\kappa}\left(\frac{1}{\kappa}-1\right) y^{1-2 \kappa / \kappa}\right)<0
$$

we are guaranteed to have a unique maximizer of $h_{S}$ on $[0, \bar{y}]$

$$
y_{\mathrm{b}}^{0}=\min \left\{y: y^{1-\kappa}(\beta+y)^{2 \kappa}=k \text { or } y=\bar{y}\right\}
$$




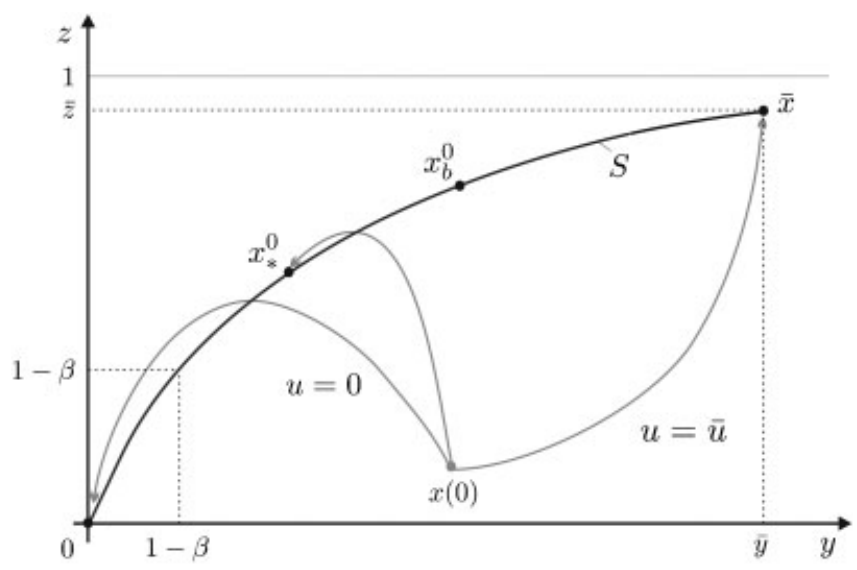

Figure 1 . Set of stationary states $S$, including the best equilibrium state $x_{\mathrm{b}}^{0}$ and optimal steady state $x_{*}^{0}$.

where $k:=(\kappa \gamma \beta)^{\kappa} / \alpha$. Thus $z_{\mathrm{b}}^{0}=y_{\mathrm{b}}^{0} /\left(\beta+y_{\mathrm{b}}^{0}\right)$ is also uniquely determined, and

$$
x_{\mathrm{b}}^{0}=\left(y_{\mathrm{b}}^{0}, \frac{y_{\mathrm{b}}^{0}}{\beta+y_{\mathrm{b}}^{0}}\right)
$$

\subsection{Asymptotic behaviour and optimal steady state $x_{*}^{0}$}

Consider a constant admissible pair $\left(x_{*}^{0}, u_{*}^{0}\right) \in S \times U$, with $x_{*}^{0}=\left(y_{*}^{0}, z_{*}^{0}\right)^{\prime}$, that satisfies the necessary optimality conditions in Proposition 2. Then, according to (20)-(21), we have

$$
\begin{gathered}
\dot{\lambda}=\alpha \lambda-\mu\left(1-z_{*}^{0}\right) \\
\dot{\mu}=-\gamma \mathrm{e}^{-\rho t}+\mu\left(\beta+y_{*}^{0}\right)
\end{gathered}
$$

Furthermore, the system equations (1)-(2) give

$$
\begin{gathered}
0=\left(u_{*}^{0}\right)^{\kappa}-\alpha y_{*}^{0} \\
0=\left(1-z_{*}^{0}\right) y_{*}^{0}-\beta z_{*}^{0}
\end{gathered}
$$

With Proposition 2, parts (ii) and (iii), and using (19), the optimal control $u_{*}^{0}$ is of the form,

$$
u_{*}^{0}=\min \left\{\bar{u},\left(\kappa \lambda \mathrm{e}^{\rho t}\right)^{1 / 1-\kappa}\right\}
$$


From (46) we know that

$$
\mu(t)=\left(\mu(0)-\frac{\gamma}{\rho+\beta+y_{*}^{0}}\right) \mathrm{e}^{\left(\beta+y_{*}^{0}\right) t}+\frac{\gamma}{\rho+\beta+y_{*}^{0}} \mathrm{e}^{-\rho t}
$$

so that by Proposition 2, part (iii), and Proposition 3 necessarily $\mu(0)=\gamma /\left(\rho+\beta+y_{*}^{0}\right)$. Substituting the resulting expression for $\mu(t)$ into (51) we have

$$
\dot{\lambda}=\alpha \lambda-\mu\left(1-z_{*}^{0}\right)=\alpha \lambda-\frac{\gamma\left(1-z_{*}^{0}\right)}{\rho+\beta+y_{*}^{0}} \mathrm{e}^{-\rho t}
$$

whence with (45)

$$
\lambda(t)=\left(\lambda(0)-\frac{\gamma\left(1-z_{*}^{0}\right)}{(\rho+\alpha)\left(\rho+\beta+y_{*}^{0}\right)}\right) \exp \left(\frac{\gamma\left(1-z_{*}^{0}\right) t}{\rho+\beta+y_{*}^{0}}\right)+\frac{\gamma\left(1-z_{*}^{0}\right) \mathrm{e}^{-\rho t}}{(\rho+\alpha)\left(\rho+\beta+y_{*}^{0}\right)}
$$

As before $\lambda(0)$ is determined uniquely by the necessary optimality conditions, so that we obtain

$$
\lambda(t)=\frac{\gamma\left(1-z_{*}^{0}\right) \mathrm{e}^{-\rho t}}{(\rho+\alpha)\left(\rho+\beta+y_{*}^{0}\right)}, \quad \mu(t)=\frac{\gamma \mathrm{e}^{-\rho t}}{\rho+\beta+y_{*}^{0}}
$$

Rewriting (55) it is then

$$
u_{*}^{0}=\min \left\{\bar{u},\left(\frac{\kappa \gamma\left(1-z_{*}^{0}\right)}{(\rho+\alpha)\left(\rho+\beta+y_{*}^{0}\right)}\right)^{1 / 1-\kappa}\right\}
$$

and thus, using (53)-(54)

$$
y_{*}^{0}=\min \left\{y: y^{1-\kappa}(\rho+\beta+y)^{\kappa}(\beta+y)^{\kappa}=k_{*} \text { or } y=\bar{y}\right\}
$$

where we have set

$$
k_{*}:=\frac{(\kappa \gamma \beta)^{\kappa}}{\alpha^{1-\kappa}(\rho+\alpha)^{\kappa}}
$$

The following proposition summarizes these results.

Proposition 4 (Optimal steady state)

Let $\left(x_{*}, u_{*}\right)$ be an optimal pair for $(P)$. If

$$
\left(x_{*}(t), u_{*}(t)\right)=\left(x_{*}(0), u_{*}(0)\right) \text { for all } t \in \mathbb{R}_{+}
$$

then $\left(x_{*}, u_{*}\right)=\left(x_{*}^{0}, u_{*}^{0}\right)$, where $u_{*}^{0}$ is given by $(56)$, and $x_{0}^{*}=\left(y_{*}^{0}, y_{*}^{0} /\left(\beta+y_{*}^{0}\right)\right)$ with $y_{*}^{0}$ determined by (57).

\section{Remark}

(a) We refer to a constant optimal pair $\left(x_{*}^{0}, u_{*}^{0}\right)$ as an optimal steady state (or more precisely: optimal steady state-control tuple). We have shown above that there is only one 
candidate for optimal steady state that satisfies the necessary optimality conditions of Proposition 2. Optimality of that state will be a consequence of Proposition 6 below.

(b) Comparing (49) with (57) and noting $k_{*}<k$, it follows that $0<x_{*}^{0}<x_{\mathrm{b}}^{0}$ (see Figures 2 and 3). In other words, $x_{*}^{0}$ lies in $S$ between the origin and the best equilibrium state $x_{\mathrm{b}}^{*}$.

(c) Note that as $\rho$ tends to zero from above, one obtains the best equilibrium state as a limit, i.e.

$$
\lim _{\rho \rightarrow 0+} x_{*}^{0}=\lim _{\rho \rightarrow 0+} x_{\mathrm{b}}^{0}
$$

since $\lim _{\rho \rightarrow 0+} k_{*}=k$ (see Figure 3).

(d) From (53)-(54) and (56) we can immediately conclude that

$$
\lim _{\rho \rightarrow \infty} x_{*}^{0}=0
$$

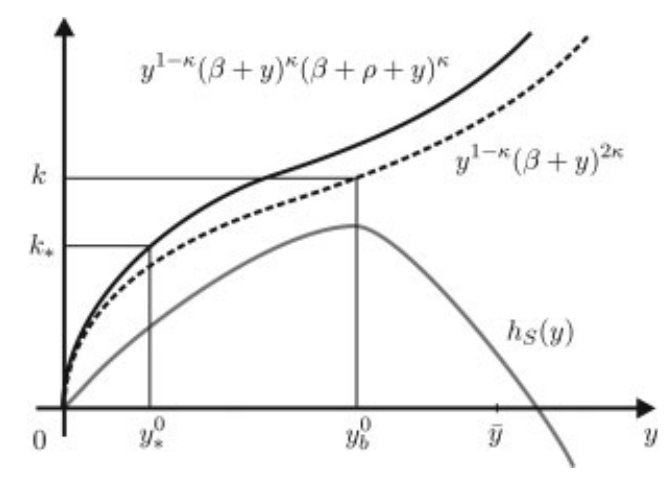

Figure 2. Illustration of the first-order conditions for determining $y_{*}^{0}<y_{\mathrm{b}}^{0}<\bar{y}$.

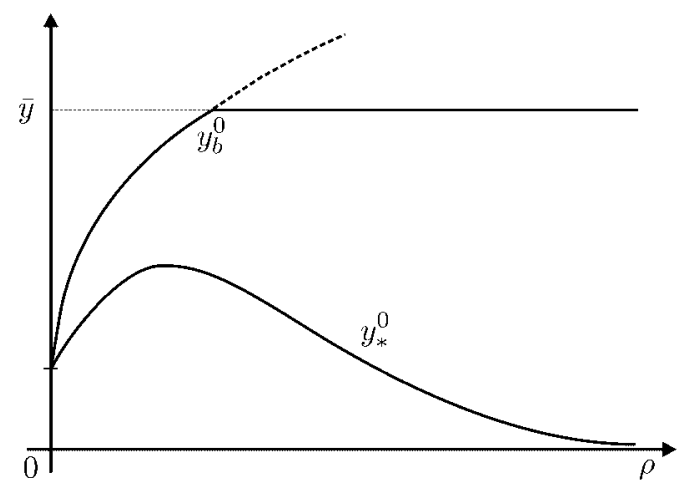

Figure 3. Comparison of $y_{*}^{0}$ and $y_{\mathrm{b}}^{0}$ as a function of $\rho$. 
Therefore for $\rho$ large enough, the optimal steady state $x_{*}^{0}$ will be an interior point of $S \subset X$.

(e) It is possible to obtain the optimal steady state $x_{*}^{0}=\left(y_{*}^{0}, z_{*}^{0}\right)$ and associated equilibrium control $u_{*}^{0}$ as the solution of Feinstein and Luenberger's [12] 'implicit programming problem,' $\left(x_{*}^{0}, u_{*}^{0}\right)=\arg \max _{((y, z), u) \in X \times U} h(z, u)$, subject to $u^{\kappa}-\alpha y=\rho\left(y-y_{*}^{0}\right)$ and $(1-$ $z) y-\beta z=\rho\left(z-z_{*}^{0}\right)$. In this paper we have opted for a (computationally equivalent) direct method to find $x_{*}^{0}$, since it employs the Hamiltonian system which is needed for our formulation of an infinite-horizon maximum principle and is used in the discussion of asymptotic stability below.

The optimal steady state-control tuple $\left(x_{*}^{0}, u_{*}^{0}\right)$ is unique. However, it is not an easy task to guarantee that any optimal trajectory asymptotically converges towards that state, since the Hamiltonian is not convex-concave. Also, more delicate results for non-convex systems, such as the ones by Rockafellar [13] (reported in Reference [12]) or more generally Haurie [33], may be unsuitable, for they rely on convexity-concavity of the (maximized) Hamiltonian in a neighbourhood of the optimal steady state or the existence of ' $G$ supported trajectories' (where $G(x)$ corresponds to the set of system velocities at state $x$ ), respectively.

To investigate the local behaviour around the optimal steady state $x_{*}^{0}$, let us first rewrite the Hamiltonian system in current-value form. For this we set $\lambda_{\mathrm{cv}}:=\lambda \mathrm{e}^{\rho t}$ and $\mu_{\mathrm{cv}}:=\mu \mathrm{e}^{\rho t}$, and obtain an equivalent set of autonomous equations

$$
\begin{gathered}
\dot{y}=\min \left\{\bar{u}^{\kappa},\left(\kappa \lambda_{\mathrm{cv}}\right)^{\kappa / 1-\kappa}\right\}-\alpha y \\
\dot{z}=(1-z) y-\beta z \\
\dot{\lambda}_{\mathrm{cv}}=(\rho+\alpha) \lambda_{\mathrm{cv}}-\mu_{\mathrm{cv}}(1-z) \\
\dot{\mu}_{\mathrm{cv}}=-\gamma+\mu_{\mathrm{cv}}(\rho+\beta+y)
\end{gathered}
$$

Note that the unique equilibrium point of this system is $\left(y_{*}^{0}, z_{*}^{0}, \lambda_{\mathrm{cv}}^{0}, \mu_{\mathrm{cv}}^{0}\right)^{\prime}$, where

$$
\lambda_{\mathrm{cv}}^{0}=\frac{\gamma\left(1-z_{*}^{0}\right)}{(\rho+\alpha)\left(\rho+\beta+y_{*}^{0}\right)}, \quad \mu_{\mathrm{cv}}^{0}=\frac{\gamma}{\rho+\beta+y_{*}^{0}}
$$

and $\left(y_{*}^{0}, z_{*}^{0}\right)$ as determined earlier. To avoid non-smooth behaviour in the neighbourhood of the equilibrium point, it is sufficient to assume that the optimal steady state $x_{*}^{0}$ is an interior point of the set of all possible steady states $S .^{17}$

\footnotetext{
$\overline{{ }^{17} \text { This implies that }}\left(\kappa \lambda_{\mathrm{cv}}\right)^{1 / 1-\kappa}<\bar{u}$ for all $\lambda_{\mathrm{cv}}$ in a neighbourhood of $\lambda_{\mathrm{cv}}^{0}$, whence the smoothness of the system (60)-(63) in a neighbourhood of $\left(x_{*}^{0}, \psi_{\mathrm{cv}}^{0}\right)$.
} 


\section{Assumption 1}

The optimal steady state $x_{*}^{0}$ lies in the interior of $S$, i.e. $x_{*}^{0}<\bar{x}$.

\section{Remark}

It is clear that Assumption 1 is weaker than (28), since it does not impose global restrictions on the magnitude of $u_{*}$ for instance.

To shift the unique equilibrium of our Hamiltonian system to the origin, we introduce new co-ordinates $\phi=\left(\phi_{1}, \phi_{2}, \phi_{3}, \phi_{4}\right)^{\prime}$,

$$
\phi:=\left[\begin{array}{c}
y-y_{*}^{0} \\
z-z_{*}^{0} \\
\lambda-\lambda_{\mathrm{cv}}^{0} \\
\mu-\mu_{\mathrm{cv}}^{0}
\end{array}\right]
$$

so that (60)-(63) can be written equivalently as a perturbed linear system

$$
\dot{\phi}=A \phi+f(\phi)
$$

where

$$
A=\left[\begin{array}{cccc}
-\alpha & 0 & l_{1} & 0 \\
1-z_{*}^{0} & -\left(\beta+y_{*}^{0}\right) & 0 & 0 \\
0 & \mu_{\mathrm{cv}}^{0} & \rho+\alpha & -\left(1-z_{*}^{0}\right) \\
\mu_{\mathrm{cv}}^{0} & 0 & 0 & \rho+\beta+y_{*}^{0}
\end{array}\right], \quad f(\phi)=\left[\begin{array}{c}
l_{2} \phi_{3}^{2}+O\left(\phi_{3}^{3}\right) \\
-\phi_{1} \phi_{2} \\
-\phi_{2} \phi_{4} \\
\phi_{1} \phi_{4}
\end{array}\right]
$$

and

$$
l_{0}=\alpha y_{*}^{0}, \quad l_{1}=\frac{\kappa}{1-\kappa} \frac{\left(\kappa \lambda_{\mathrm{cv}}^{0}\right)^{\kappa / 1-\kappa}}{\lambda_{\mathrm{cv}}^{0}}, \quad l_{2}=\frac{\kappa(2 \kappa-1)}{2(1-\kappa)^{2}} \frac{\left(\kappa \lambda_{\mathrm{cv}}^{0}\right)^{\kappa / 1-\kappa}}{\left(\lambda_{\mathrm{cv}}^{0}\right)^{2}}
$$

are the first terms in the Taylor series expansion

$$
\left(\kappa\left(\phi_{3}+\lambda_{\mathrm{cv}}^{0}\right)\right)^{\kappa / 1-\kappa}=\sum_{k=0}^{\infty} l_{k} \phi_{3}^{k}
$$

around $\phi_{3}=0$. We note that the eigenvalues $a_{1}, a_{2}, a_{3}, a_{4}$ of the system matrix $A$ are all distinct, two of them $\left(a_{1}, a_{2}\right)$ with negative real part and the other two $\left(a_{3}, a_{4}\right)$ with positive real part. They are given by

$$
\begin{aligned}
& a_{1 / 2}=\frac{\rho}{2}-\sqrt{(\rho+\alpha)^{2}+2\left(\rho+\beta+y_{*}^{0}\right)\left(\beta+y_{*}^{0}\right)+\alpha^{2} \pm 2 \sqrt{\Delta}} \\
& a_{3 / 4}=\frac{\rho}{2}+\sqrt{(\rho+\alpha)^{2}+2\left(\rho+\beta+y_{*}^{0}\right)\left(\beta+y_{*}^{0}\right)+\alpha^{2} \pm 2 \sqrt{\Delta}}
\end{aligned}
$$


where we have set

$$
\begin{aligned}
\Delta:= & \left(y_{*}^{0}\right)^{4}+2(\rho+2 \beta)\left(y_{*}^{0}\right)^{3}+\left(\rho(\rho-2 \alpha)+6 \beta(\rho+\beta)-2 \alpha^{2}\right)\left(y_{*}^{0}\right)^{2} \\
& +2\left((\beta-\alpha) \rho^{2}+\left(4 \beta^{2}-(\alpha+\beta)^{2}\right) \rho+2 \beta\left(\alpha^{2}+\beta^{2}\right)-4 l_{1} \mu_{\mathrm{cv}}^{0}\left(1-z_{*}^{0}\right)\right) y_{*}^{0} \\
& +(\alpha-\beta)^{2} \rho^{2}+2\left((\alpha+\beta)(\beta-\alpha)^{2}-2 l_{1} \mu_{\mathrm{cv}}^{0}\left(1-z_{*}^{0}\right)\right) \rho+\left(\beta^{2}-\alpha^{2}\right)^{2}-8 l_{1} \mu_{\mathrm{cv}}^{0}\left(1-z_{*}^{0}\right)
\end{aligned}
$$

Next we would like to find a similarity transform $\mathscr{S}$ that brings the linear part $A$ of system (65) into the Jordan form $\mathscr{J}=\mathscr{S}^{-1} A \mathscr{S}$. The resulting (block-) diagonal system matrix $\mathscr{J}$ allows us, provided the perturbation function satisfies a Lipschitz condition, to guarantee the convergence of trajectories to the optimal steady state (namely the origin in the transformed co-ordinates). Given an appropriate similarity transform $\mathscr{S}$ that brings $A$ into Jordan form, we introduce (following Reference [14, pp. 68-69]) new co-ordinates $\varphi=(\xi, \eta)^{\prime}$ such that

$$
\phi=\mathscr{S} \varphi, \quad \operatorname{det} \mathscr{S} \neq 0
$$

Then (65) can be written in the new co-ordinates,

$$
\dot{\varphi}=\mathscr{J} \varphi+\mathscr{S}^{-1} f(\mathscr{S} \varphi), \quad J=\mathscr{S}^{-1} A \mathscr{S}
$$

The transformed system (68) is, however, not properly defined in all cases. In particular, the eigenvalues of $A$ may be complex, whence complex entries in $\mathscr{S}$ may render the expression $f(\mathscr{S} \varphi)$ meaningless. In our case, we can see from (66)-(67) that either all four eigenvalues $a_{1}, \ldots, a_{4}$ are real, or they form two conjugate pairs $a_{1 / 2}$ and $a_{3 / 4}$. In the latter case we follow Reference [14, p. 69], introducing the matrix

$$
\mathscr{S}_{0}=\left[\begin{array}{cc}
I & \mathrm{i} I \\
I & -\mathrm{i} I
\end{array}\right]
$$

where $I$ is the $2 \times 2$ identity and $\mathrm{i}=\sqrt{-1}$. Then the change of variables $\phi=\mathscr{S}_{0} w$ transforms (68) into the real system

$$
\dot{w}=\mathscr{S}_{0}^{-1} \mathscr{J} \mathscr{S}_{0} w+\mathscr{S}_{0}^{-1} \mathscr{S}^{-1} f\left(\mathscr{S} \mathscr{S}_{0} w\right)
$$

or equivalently, by taking linear combinations with constant coefficients 1 or $\pm \mathrm{i}$

$$
\left(\dot{\mathscr{S}}_{0} w\right)=\mathscr{J}\left(\mathscr{S}_{0} w\right)+\mathscr{S} f\left(\mathscr{S}\left(\mathscr{S}_{0} w\right)\right)
$$

Thus, interpreting (68) if necessary as (69) we can write the perturbed system in the blockdiagonal form

$$
\begin{aligned}
& \dot{\xi}=P \xi+F_{1}(\xi, \eta) \\
& \dot{\eta}=Q \eta+F_{2}(\xi, \eta)
\end{aligned}
$$

where (in case $a_{1}, \ldots, a_{4}$ are real) $P:=\operatorname{diag}\left\{a_{1}, a_{2}\right\}, Q:=\operatorname{diag}\left\{a_{3}, a_{4}\right\}$, and $\left(F_{1}, F_{2}\right)^{\prime}=$ $\mathscr{S}^{-1} f(\mathscr{S} \varphi)$ which contains only higher powers of $\varphi=(\xi, \eta)^{\prime}$. The following proposition by 
Hartman [14] allows us to conclude about the asymptotic behaviour near the optimal steady state

Proposition 5 (Asymptotic integration; Hartman [14, pp. 294-296])

Let a system of differential equations have the form (70)-(71), where

(i) the eigenvalues $p_{1}, p_{2}$ and $q_{1}, q_{2}$ of $P$ and $Q$ satisfy

$$
\operatorname{Re}\left(p_{j}\right) \leqslant \sigma, \quad \operatorname{Re}\left(q_{k}\right)>\sigma
$$

for some real $\sigma<0$;

(ii) $F=\left(F_{1}, F_{2}\right)$ is continuous and satisfies

$$
\frac{\|F(\varphi)\|}{\|\varphi\|} \rightarrow 0, \quad \text { as }(t, \varphi) \rightarrow(\infty, 0) \quad\left(\varphi=(\xi, \eta)^{\prime}\right)
$$

Then the following conclusions hold:

(A) There exist $T>0$ and $\delta>0$ such that for every $t_{0}>T$ and $\xi_{0}$ satisfying $\left\|\xi_{0}\right\|<\delta$ there is a $\eta_{0}$ such that the initial value problem (70)-(71) with

$$
\xi\left(t_{0}\right)=\xi_{0}, \quad \eta\left(t_{0}\right)=\eta_{0}
$$

has a solution for $t \geqslant t_{0}$ satisfying either $\varphi(t)=(\xi(t), \eta(t)) \equiv 0$ or $\xi(t) \neq 0$ for $t \geqslant t_{0}$, and

$$
\begin{gathered}
\|\eta(t)\|=o(\|\xi(t)\|) \quad \text { as } t \rightarrow \infty \\
\limsup _{t \rightarrow \infty} \frac{\log \|\varphi(t)\|}{t} \leqslant \sigma
\end{gathered}
$$

(B) If in addition, $F$ satisfies

$$
\frac{\left\|F\left(\varphi^{1}\right)-F\left(\varphi^{2}\right)\right\|}{\left\|\varphi^{1}-\varphi^{2}\right\|} \rightarrow 0 \quad \text { as }\left(t, \varphi^{1}, \varphi^{2}\right) \rightarrow(\infty, 0,0)
$$

when $\varphi^{1} \neq \varphi^{2}$, then there exists a small $\delta_{0}>0$ with the property that if $t_{0}$ is sufficiently large and $\left\|\xi_{0}\right\|$ is sufficiently small, there is a unique $\eta_{0}=g\left(t_{0}, \xi_{0}\right)$ such that the solution $\varphi(t)=(\xi(t), \eta(t))^{\prime}$ of the above initial value problem exists and satisfies $\|\varphi(t)\|<\delta$ for $t \geqslant 0$. Furthermore, the function $g\left(t_{0}, \xi_{0}\right)$ is of the same smoothness as $F$.

\section{Remark}

All the hypotheses of Proposition 6 are evidently satisfied, by construction. In particular, $F$ is clearly analytic in the neighbourhood of the origin, so that $g$ is at least infinitely differentiable.

To exclude limit cycles at least locally, Proposition 6 per se is not sufficient, as for a given $x_{0}$ there might be two different initial conditions $\psi_{0}^{1} \neq \psi_{0}^{2}$, such that the associated state trajectory 
converges to $x_{*}^{0}$ for $\psi(0)=\psi_{0}^{1}$ and to a limit cycle for $\psi(0)=\psi_{0}^{2}$. If the optimal trajectory is unique, such behaviour is not possible.

Assumption 2 (Uniqueness of optimal trajectories)

For any given initial condition $x_{0}$, the optimal pair $\left(x_{*}, u_{*}\right)$ for $(P)$ is unique.

\section{Remark}

In Remark (c) after Proposition 2 we pointed out that the (maximized) Hamiltonian for $(P)$ is not concave in the state variable, and thus standard uniqueness results, such as the one provided by Mangasarian [34], and the generalization thereof for infinite-horizon problems by Arrow and Kurz [18] do not hold. In our particular problem $(P)$ it is possible to use the concavity in $u$ of the Hamilton-Pontryagin function together with the maximality condition in Proposition 2 to guarantee the uniqueness of an optimal trajectory for every specific initial condition $\left(x_{0}, \psi_{0}\right)$. However, since $\psi_{0}$ by (43)-(44) in turn depends on the optimal trajectory, ensuring uniqueness remains a non-trivial task; Assumption 2 is therefore restrictive and needs to be proved or disproved as a property of the system.

We are now able to provide our result on local asymptotic convergence of any optimal trajectory starting close enough to $x_{*}^{0}$.

\section{Proposition 6 (Local asymptotic convergence)}

If assumptions 1 and 2 hold, then there is a $\delta>0$ such that for every $x_{0} \in X$ with $\left\|x_{0}-x_{0}^{*}\right\|<\delta$, the optimal trajectory $x_{*}(t)$ with respect to $(P)$ converges to the optimal steady state $x_{*}^{0}$ as $t \rightarrow \infty$.

\section{Proof}

Given $x_{0}$ in a small enough neighbourhood of $x_{*}^{0}$, Proposition 5 guarantees the existence of a unique initial condition $\psi(0)=\psi_{0}$ such that $\left(x_{*}(t), \psi(t) \mathrm{e}^{\rho t}\right) \rightarrow\left(x_{*}^{0}, \psi_{\mathrm{cv}}^{0}\right)$, where $\psi_{\mathrm{cv}}^{0}=\left(\lambda_{\mathrm{cv}}^{0}, \mu_{\mathrm{cv}}^{0}\right)^{\prime}$, as determined previously in (64). Assumption 2 ensures the uniqueness of the optimal pair, and thus by Proposition 3 there cannot be any other trajectory $(x(t), \psi(t))$ of the Hamiltonian system (60)-(63) that satisfies the necessary optimality conditions of Proposition 2. Indeed, $\lim _{t \rightarrow \infty} \psi(t)=0$ if and only if $\psi(0)=(\lambda(0), \mu(0))^{\prime}$ is given by (43)-(44). Hence, it is indeed the (by Assumption 2 unique) optimal trajectory $x_{*}(t)$ (not any other trajectory of the Hamiltonian system) that converges to $x_{*}^{0}$ as $t \rightarrow \infty$. This concludes the proof.

\section{Remark}

(a) Proposition 6 implies the optimality of $x_{*}^{0}$. To see this, it is enough to take any particular optimal trajectory $x_{*}(t)$ (unique by Assumption 2) that converges to $x_{*}^{0}$, and then consider the sequence of problems with initial conditions $x_{*}\left(T_{k}\right)$, where $T_{k}<T_{k+1} \rightarrow \infty$ as $k \rightarrow \infty$. For each $k$ the optimal solution $x_{k}$ satisfies $x_{k}(t)=x_{*}\left(t+T_{k}\right)$ by the optimality principle, so that in the $\operatorname{limit}_{\lim _{k \rightarrow \infty}} x_{k}(t)=x_{*}^{0}$ strongly. ${ }^{18}$

\footnotetext{
${ }^{18}$ Indeed, for any $\varepsilon>0$ there is a $T(\varepsilon)>0$ such that $\left\|x_{*}(t)-x_{*}^{0}\right\|<\varepsilon$ for all $t \geqslant T(\varepsilon)$. Thus, considering a sequence $\varepsilon_{k}>0, k=1,2, \ldots$, with $\varepsilon_{k} \rightarrow 0$ and $T\left(\varepsilon_{k}\right)=: T_{k} \rightarrow \infty$ (w.l.o.g. $T_{k+1}>T_{k}$ ), as $k \rightarrow \infty$, it is $\left\|x_{k}(t)-x_{*}^{0}\right\| \leqslant \varepsilon_{k}$ for all $t \geqslant 0$ and $k \geqslant 1$. Hence $x_{k}(t) \rightarrow x_{*}^{0}$ uniformly on $\mathbb{R}_{+}$as $k \rightarrow \infty$.
} 
(b) Assume that there exists a (global) limit cycle $L$ that is an optimal trajectory, i.e. there is an optimal pair $\left(x_{*}, u_{*}\right)$ for $(P)$ such that

$$
x_{*}(0) \in L \Leftrightarrow x_{*}(t) \in L \quad \text { for all } t \in \mathbb{R}_{+}
$$

and there is a finite $T>0$ such that

$$
x_{*}(t)=x_{*}(t+n T) \text { for all } n \in \mathbb{N}, t \in[0, T)
$$

Consequently, $u_{*}$ needs to be also $T$-periodic, i.e.

$$
u_{*}(t)=u_{*}(t+n T) \text { for all } n \in \mathbb{N}, t \in[0, T)
$$

Let $\psi=(\lambda, \mu)^{\prime}$ be an adjoint variable satisfying the necessary optimality conditions of Proposition 2 . The $T$-periodicity of the state trajectory (77) implies the $T$-periodicity of $\lambda_{\mathrm{cv}}(t)$ and $\mu_{\mathrm{cv}}(t)$, with initial conditions (from (43)-(44)) ${ }^{19}$

$$
\begin{aligned}
& \lambda_{\mathrm{cv}}(0)=\frac{1}{1-\mathrm{e}^{-\rho T}} \int_{0}^{T} \mathrm{e}^{-\rho t} \mu(t)\left(1-z_{*}(t)\right) \mathrm{d} t \\
& \mu_{\mathrm{cv}}(0)=\frac{\int_{0}^{T} \exp \left(-(\rho+\beta) t-\int_{0}^{t} y_{*}(\theta) \mathrm{d} \theta\right) \mathrm{d} t}{1-\exp \left(-(\rho+\beta) T-\int_{0}^{T} y_{*}(\theta) \mathrm{d} \theta\right)}
\end{aligned}
$$

In addition, we know that $L \subset X$ (if it exists) must be oriented positively around $x_{*}^{0}$, and by Assumption 2 cannot intersect itself. Indeed if there were any intersection points an optimal trajectory starting at such an intersection would not be unique. $L$ denotes the projection of a one-dimensional limit cycle $C \subset X \times \mathbb{R}_{+}^{2}$ onto $X$, which thus in principle could exhibit intersections, even though $C$ possesses none. Thus, if it can be shown that for the particular initial condition $x_{0}=0$ the optimal trajectory $x_{*}(t)$ with respect to $(P)$ converges to $x_{*}^{0}$, then there cannot exist any limit cycle. ${ }^{20}$

(c) If $x_{*}^{0}=\bar{x}$, i.e. if Assumption 1 does not hold, then there cannot exist a (non-trivial) limit cycle. This follows directly from (b). Hence in this case, we have even global asymptotic convergence of any optimal trajectory $x_{*}(t)$ to $x_{*}^{0}$ as $t \rightarrow \infty$. Moreover, this global result does not depend on Assumption 2 at all. Economically this corresponds to the situation of a tight restriction on the rate of advertising spending ( $\bar{u}$ small), so that it is essentially optimal to spend as much as possible on advertising in order to maximize discounted profits.

\section{DISCUSSION}

Having a complete picture of an optimal policy is very important for any decision maker, allowing her to simplify decision rules and implement feedback that moves her system along an

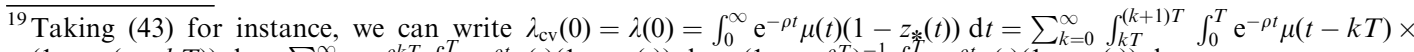
$\left(1-z_{*}(t-k T)\right) \mathrm{d} t=\sum_{k=0}^{\infty} \mathrm{e}^{-\rho k T} \int_{0}^{T} \mathrm{e}^{-\rho t} \mu(t)\left(1-z_{*}(t)\right) \mathrm{d} t=\left(1-\mathrm{e}^{-\rho T}\right)^{-1} \int_{0}^{T} \mathrm{e}^{-\rho t} \mu(t)\left(1-z_{*}(t)\right) \mathrm{d} t$.

${ }^{20}$ For a particular parameter vector $(\alpha, \beta, \gamma, \kappa, \rho, \bar{u})$ a 'proof' that there is asymptotic convergence towards $x_{*}^{0}$ can thus be obtained via numerical methods. Of course this assumes that in the spirit of LaSalle's theorem (Theorem 3.4 in Reference [35, p. 117], a Lyapunov function $V(x)$ has been found that decreases along any optimal trajectory and can stay constant only on an optimal limit cycle or the optimal steady state, so that asymptotically any optimal trajectory needs to converge towards either a limit cycle or the optimal steady state.
} 
optimal path. In addition, the assertion of global asymptotic convergence of optimal trajectories enables the decision maker, instead of constantly attempting to measure the value of her objective function, to concentrate on steering the system to the optimal steady state in an efficient manner. We have laid the groundwork to provide such a complete picture (relating, for instance, optimal trajectories to initial points of the state space) for the problem of maximizing discounted profits as a response to advertising for and sales of durable goods, based on a combination of classic models by Vidale-Wolfe [26] and Nerlove-Arrow [24].

In particular, we have analysed the synthesis and asymptotic behaviour of an optimal infinitehorizon advertising spending policy. An infinite-horizon formulation of the optimal advertising problem naturally represents a 'going concern' for the promotion of durable products in a fixedprice environment. To obtain necessary optimality conditions that allow the effective selection of candidates for an optimal policy, it is possible (using the method of smooth approximation) to construct a version of the Pontryagin Maximum Principle (Proposition 2) that includes growth conditions, stronger than the 'natural' transversality conditions proposed by Arrow and Kurz [18]. In addition, we find the (unique) optimal steady state $x_{*}^{0}$, which generally does not coincide with the global static maximizer $x_{\mathrm{b}}^{0}$ of current-value profits over all equilibrium states. This is because starting from, say, the best equilibrium state $x_{\mathrm{b}}^{0}$, it may be optimal for the decision maker to steer the system first to some unsustainable but more profitable states before reaching $x_{*}^{0}$. Indeed, such a policy is always optimal when starting close enough to the optimal steady state and it is optimal for any initial state if there is no limit cycle. Limit cycles can be excluded for a certain subset of parameters corresponding to an 'underfunded' situation with a too restrictive upper bound $\bar{u}$ on the advertising spending rate $u$. If in addition, for a given parameter vector $(\alpha, \beta, \gamma, \kappa, \rho, \bar{u})$ it can be shown numerically (with an appropriate bound on errors) that the optimal trajectory tends from $x_{0}=0$ to $x_{*}^{0}$, then the optimal trajectory from any initial state $x_{0} \in X$ must also converge to $x_{*}^{0}$.

Non-linear infinite-horizon optimal control problems with non-convex Hamiltonian, such as the one considered here, arise frequently in economics, such as, e.g. in optimal advertising or, more generally, in optimal product diffusion problems. Results on the asymptotic convergence of optimal trajectories typically available in the literature require strong curvature properties of the Hamiltonian that are not satisfied for non-convex systems. Even results based on sufficient optimality conditions available for certain classes of non-convex systems such as in Reference [33] do not appear useful in our context, since they need strong assumptions on the global system behaviour outside a neighbourhood of the optimal equilibrium state. Our approach is a local one, and the growth condition of Proposition 2 part (iii) helps ensure asymptotic convergence of the Hamiltonian system of differential equations towards the optimal equilibrium state. Most results obtained in this paper can be expected to hold for a larger class of systems (including, for instance, price as a decision variable) that preserve the existence of a compact invariant set which is reached in finite time by all trajectories.

\section{APPENDIX A: REACHABILITY AND TIME-OPTIMAL CONTROL}

In this appendix, we discuss reachability and the related problem of steering the system from an initial state to a terminal state within the set of reachable states. It turns out that for each initial state the set of reachable states contains an invariant subset, independent of the initial state. In principle, those are the states on which to focus discussion, and only they should provide 
plausible initial conditions, unless there has been a switch in the modelling conditions since the inception of the product. Such a switch may occur as, e.g. product diffusion models typically contain additional terms (see e.g. References [36,37]), for instance a 'word-of-mouth effect,' which might change and/or become ineffective over time.

\section{A.1. Reachability}

We are interested in obtaining an expression for the set of all states $\mathscr{R}\left(x^{\mathrm{i}}\right)$ that can be reached from a given initial state $x^{\mathrm{i}}$. Let us first consider the trajectories passing through a given state $(\hat{y}, \hat{z})$ in the interior of $X$ applying a constant control $u^{0} \in U$. From the system (1)-(2) we obtain, using the abbreviation $v^{0}=\left(u^{0}\right)^{\kappa}$,

$$
\frac{\mathrm{d} z(y)}{\mathrm{d} y}=\frac{(1-z) y-\beta z}{v^{0}-\alpha y}
$$

provided that $v^{0} \neq \alpha y$. The (unique) solution of (A1) with initial condition

$$
z(\hat{y})=\hat{z}
$$

is then given by

$$
z(y)=\hat{z} \mathrm{e}^{(y-\hat{y}) / \alpha}\left(\frac{v^{0}-\alpha y}{v^{0}-\alpha \hat{y}}\right)^{\alpha \beta+v^{0} / \alpha^{2}}+\int_{\hat{y}}^{y}\left(\frac{v^{0}-\alpha y}{v^{0}-\alpha s}\right)^{\alpha \beta+v^{0} / \alpha^{2}} \frac{s \mathrm{e}^{(y-s) / \alpha}}{v^{0}-\alpha s} \mathrm{~d} s
$$

From (48) and (A1) we note that

$$
\frac{\mathrm{d} z(y)}{\mathrm{d} y}=0 \Leftrightarrow(y, z(y)) \in S
$$

or more precisely

$$
\operatorname{sgn}\left(\frac{\mathrm{d} z(y)}{\mathrm{d} y}\right)=\operatorname{sgn}\left(\frac{y}{\beta+y}-z(y)\right)
$$

which means that all trajectories 'above' $S$ are downward sloping, while all trajectories 'below' $S$ are upward sloping (see Figure A1). Moreover,

$$
z\left(v^{0} / \alpha\right)=\frac{v^{0}}{\alpha \beta+v^{0}}
$$

and $\left(v^{0} / \alpha, v^{0} /\left(\alpha \beta+v^{0}\right) \in S\right.$, implying that a constant control $u^{0} \in U$ moves the system (1)-(2) asymptotically to a well-specified stationary point in $S$, independent of the initial state, i.e.

$$
\lim _{t \rightarrow \infty} x(t)=\left(\frac{v^{0}}{\alpha}, \frac{v^{0}}{\alpha \beta+v^{0}}\right) \in S \quad \text { if } u(t) \equiv u^{0} \text { for } t \geqslant T
$$

Since the set of possible velocities at any point of the state-space is convex, we can limit ourselves to constant controls $u^{0} \in \partial U=\{0, \bar{u}\}$. Given an initial state $x^{\mathrm{i}} \in X$, the set of reachable 


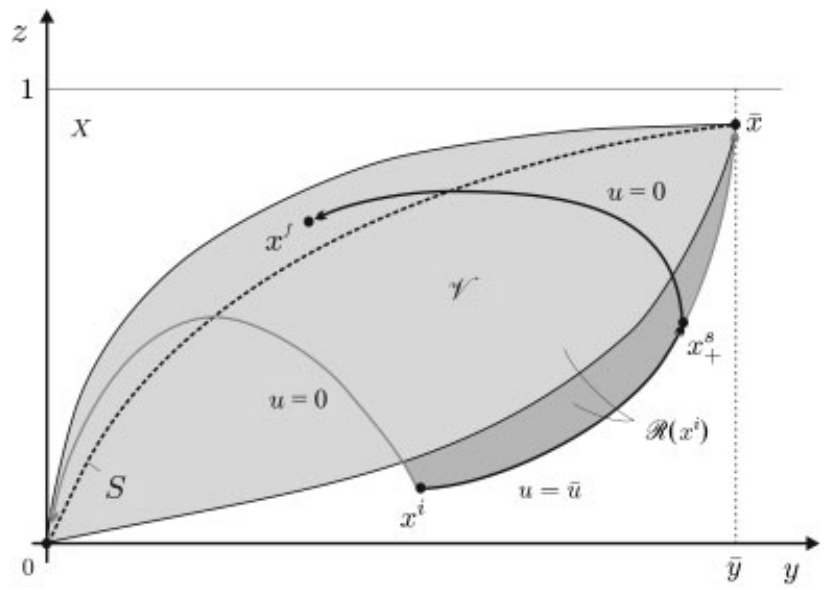

Figure A1. Time-optimal control from $x^{\mathrm{i}}$ to $x^{\mathrm{f}} \in \mathscr{R}\left(x^{\mathrm{i}}\right)$ via a single switching point $x_{+}^{s}$.

states $\mathscr{R}\left(x^{\mathrm{i}}\right)$ is described by ${ }^{21}$

$$
\mathscr{R}\left(x^{\mathrm{i}}\right)=\left\{x \in X: \zeta_{-}(y) \leqslant \min \left\{z\left(y ; x^{\mathrm{i}}, 0\right), z\left(y ; x^{\mathrm{i}}, \bar{u}\right)\right\}\right\} \cup \mathscr{V}
$$

for $z^{\mathrm{i}}>y^{\mathrm{i}} /\left(\beta+y^{\mathrm{i}}\right)$, and

$$
\mathscr{R}\left(x^{\mathrm{i}}\right)=\left\{x \in X: \max \left\{z\left(y ; x^{\mathrm{i}}, 0\right), z\left(y ; x^{\mathrm{i}}, \bar{u}\right)\right\} \leqslant \zeta_{+}\right\} \cup \mathscr{V}
$$

for $z^{\mathrm{i}} \leqslant y^{\mathrm{i}} /\left(\beta+y^{\mathrm{i}}\right)$. We have defined $\zeta_{-}(y)$ as the $z$-co-ordinate of the trajectory starting at the origin under constant control $u=\bar{u}$, and $\zeta_{+}(y)$ correspondingly as the $z$-co-ordinate of the trajectory starting at $(\bar{y}, \bar{y} /(\beta+\bar{y}))$ under constant control $u=0$. Furthermore,

$$
\mathscr{V}=\left\{x \in X: \zeta_{-}(y) \leqslant z \leqslant \zeta_{+}(y)\right\}
$$

is a compact invariant subset of the state-space for the system (1)-(2), i.e. any trajectory starting at a point in $\mathscr{V}$ is bound to stay in $\mathscr{V}$. Using (A6), we see that

$$
S \subset \mathscr{V}
$$

and any trajectory $x(t)$ starting in $X$ will eventually enter $\mathscr{V}$, i.e. there exists a time $T$, such that $x(t) \in \mathscr{V}$ for all $t \geqslant T$. In addition, the system is completely controllable in $\mathscr{V}$, and we construct a corresponding time-optimal controller that reaches any state in $\mathscr{V}$ in the shortest time possible.

\footnotetext{
${ }^{21}$ To clarify our short notation: in the description of $\mathscr{R}\left(x^{\mathrm{i}}\right), z(y)$ denotes trajectories under arbitrary controls $u \in U$ starting at $x^{\mathrm{i}} \in X$.
} 


\section{A.2. Time-optimal control}

Consider the time-optimal control problem $\left(P^{\prime}\right)$ of steering the system (1)-(2) in minimal time from an initial state $x^{\mathrm{i}} \in X$ to a final state $x^{\mathrm{f}} \in \mathscr{R}\left(x^{\mathrm{i}}\right) \backslash \partial X$ in minimal time $T<\infty$. More specifically, $\left(P^{\prime}\right)$ can be written as

$$
\left(P^{\prime}\right): \quad \int_{0}^{T}-1 \mathrm{~d} t \rightarrow \max
$$

subject to (1)-(2), (5), and

$$
x(0)=x^{\mathrm{i}}, \quad x(T)=x^{\mathrm{f}}
$$

where $T \geqslant 0$ is free, and assumed to be finite. The adjoint equations for the problem $\left(P^{\prime}\right)$ are simply the 'stationary limit' of (20)-(21)

$$
\begin{gathered}
\dot{\lambda}=\alpha \lambda-\mu(1-z) \\
\dot{\mu}=\mu(y+\beta)
\end{gathered}
$$

Maximization of the associated Hamilton-Pontryagin function yields that the optimal control for $\left(P^{\prime}\right)$ is bang-bang, i.e.

$$
u_{*}=\operatorname{bang}[0, \bar{u} ; \lambda]
$$

Since the end-time $T$ is free in this problem and subject to optimization, at the optimal time $T_{*}$ the (maximized) Hamiltonian for $\left(P^{\prime}\right)$ vanishes, i.e.

$$
H\left(T_{*}, x^{\mathrm{f}}, \psi\left(T_{*}\right)\right)=-1+\max \left\{0, \lambda\left(T_{*}\right) \bar{u}^{\kappa}\right\}-\alpha \lambda\left(T_{*}\right) y^{\mathrm{f}}+\mu\left(T_{*}\right)\left(\left(1-z^{\mathrm{f}}\right) y^{\mathrm{f}}-\beta z^{\mathrm{f}}\right)=0
$$

For the synthesis of the time-optimal controller, let us first focus on the number of possible switches. However, before we formulate and prove our result, we first of all state a well-known result from the theory of ordinary differential equations.

\section{Proposition A.1}

Any solution $u_{*}$ to the time-optimal control problem $\left(P^{\prime}\right)$ is bang-bang with at most one switch.

\section{Proof}

From (A14) it is clear that $u_{*}$ is bang-bang taking only values in $\partial U=\{0, \bar{u}\}$. Consider now the adjoint equations (A12)-(A13). Given an initial value for $\mu$, the solution of (A13) can be written according to the variation-of-constants formula as

$$
\mu(t)=\mu(0) \exp \left(\int_{0}^{t} y(\theta) \mathrm{d} \theta+\beta t\right)
$$

Note that the sign of $\mu$ does not change and depends on $\mu(0)$

$$
\operatorname{sgn}(\mu(t))=\operatorname{sgn}(\mu(0))=\text { const }
$$


Similarly, given an initial value of $\lambda$, we can employ again the variation-of-constants formula [30] and obtain an expression for $\lambda(t)$, whose changes of sign directly determine the number of switches according to (A14):

$$
\lambda(t)=\mathrm{e}^{\alpha t}\left(\lambda(0)-\int_{0}^{t} \mathrm{e}^{-\alpha \theta} \mu(\theta)(1-z(\theta)) \mathrm{d} \theta\right)
$$

Thus,

$$
\dot{\lambda}(t)=\mathrm{e}^{\alpha t}\left[\alpha\left(\lambda(0)-\int_{0}^{t} \mathrm{e}^{-\alpha \theta} \mu(\theta)(1-z(\theta)) \mathrm{d} \theta\right)-\mathrm{e}^{-\alpha t} \mu(t)(1-z(t))\right]>0
$$

if and only if

$$
\lambda(0)>\int_{0}^{t} \mu(\theta)(1-z(\theta)) \mathrm{d} \theta+\frac{\mathrm{e}^{-\alpha t}}{\alpha} \mu(t)(1-z(t))=: \varsigma(t)
$$

Consider the time derivative of the right-hand side of the last inequality,

$$
\dot{\zeta}=\mu(1-z)+\frac{\mathrm{e}^{-\alpha t}}{\alpha}\left(\frac{\mathrm{d}}{\mathrm{d} t}(\mu(1-z))-\alpha \mu(1-z)\right)
$$

and note that

$$
\frac{\mathrm{d}}{\mathrm{d} t}(\mu(1-z))=\dot{\mu}(1-z)-\mu \dot{z}=\mu(\beta+y)(1-z)-\mu((1-z) y-\beta z)=\mu
$$

using (2) and (A13). Therefore

$$
\dot{\zeta}=\mu\left[(1-z)\left(1-\mathrm{e}^{-\alpha t}\right)+\frac{\mathrm{e}^{-\alpha t}}{\alpha}\right]
$$

which implies with (A16) that

$$
\operatorname{sgn} \dot{\zeta}=\operatorname{sgn} \mu(0)=\text { const }
$$

Going back to (A17) and (A18) we remark

$$
\operatorname{sgn} \dot{\lambda}=\operatorname{sgn}(\lambda(0)-\varsigma)
$$

from which we conclude that because of the monotonicity of $\varsigma, \dot{\lambda}$ cannot change sign more than once. Therefore $\lambda$ cannot vanish more than once, so that the number of switches is at most one, which proves the proposition.

Based on Proposition A.1, we know that the final state $x^{\mathrm{f}}$ will be reached either by first applying $u=\bar{u}$ up to the switching time $\tau_{+}$, and from then on $u=0$ up to time $T_{+}$or using first $u=0$ up to the switching time $\tau_{-}$and from then on $u=\bar{u}$ up to time $T_{-}$. 


\section{ACKNOWLEDGEMENTS}

This research was supported by a David T. Morgenthaler II Faculty Scholar Award at Stanford University, the Risk Management and Decision Processes Center at the Wharton School of the University of Pennsylvania, and by the Young Scientists Summer Program at the International Institute for Applied Systems Analysis, Austria. I am indebted to Serguei Aseev for his encouragement throughout the work on this project and numerous fruitful discussions. I would also like to thank two anonymous referees, Yuri Ermoliev, Arkadii Kryazhimskii, Paul Kleindorfer, Howard Kunreuther, Alexandre Megretski, Alexandre Tarasyev, and seminar participants at the MIT Laboratory for Information and Decision Systems for helpful comments and suggestions. Of course, any remaining errors are the sole responsibility of the author.

\section{REFERENCES}

1. Sethi SP. Dynamic optimal control in advertising: a survey. SIAM Review 1977; 19(4):685-725.

2. Feichtinger G, Hartl RF, Sethi SP. Dynamic optimal control models in advertising: recent developments. Management Science 1994; 40(2):195-226.

3. Kleindorfer P, Kunreuther H. Stochastic horizons for the aggregate planning problem. Management Science 1978; 24(5):485-497.

4. Dorfman R, Samuelson PA, Solow R. Linear Programming and Economic Analysis. McGraw-Hill: New York, 1958.

5. McKenzie LW. Accumulation programs of maximum utility and the Von Neumann facet. In Value, Capital, and Growth: Papers in Honour of Sir John Hicks, Wolfe JN (ed.). Aldine: Chicago, 1968.

6. McKenzie LW. Turnpike theory. Econometrica 1976; 44(5):841-865.

7. Cass D. Optimum growth in an aggregative model of capital accumulation: a turnpike theorem. Econometrica 1996; 34(4):833-849.

8. Cass D, Shell K. The structure and stability of competitive dynamical systems. Journal of Economic Theory 1976; 12:31-70.

9. Haurie A. Optimal control on an infinite time horizon: the turnpike approach. Journal of Mathematical Economics 1976; 3:81-102.

10. Carlson DA, Haurie A. Infinite Horizon Optimal Control: Theory and Applications. Springer: Berlin, 1987.

11. Brock WA, Scheinkman J. Global asymptotic stability of optimal control systems with applications to the theory of economic growth. Journal of Economic Theory 1976; 12:164-190.

12. Feinstein CD, Luenberger DG. Analysis of the asymptotic behavior of optimal control trajectories: the implicit programming problem. SIAM Journal on Control and Optimization 1981; 19(5):561-585.

13. Rockafellar RT. Saddle points of Hamiltonian systems in convex Lagrange problems having nonzero discount rate. Journal of Economic Theory 1976; 12:71-113.

14. Hartman P. Ordinary Differential Equations. Wiley: New York, 1964.

15. Eliashberg J, Lilien GL (eds). Marketing. Elsevier Science: Amsterdam, 1993.

16. Urban GL, Hauser JR. Design and Marketing of New Products. Prentice-Hall: Englewood Cliffs, NJ, 1993.

17. Pontryagin LS, Boltyanskii VG, Gamkrelidze RV, Mishchenko EF. The Mathematical Theory of Optimal Processes. Wiley Interscience: New York, 1962.

18. Arrow KJ, Kurz M. Public Investment, The Rate of Return, and Optimal Fiscal Policy. Johns Hopkins Press: Baltimore, 1970.

19. Halkin H. Necessary conditions for optimal control problems with infinite horizons. Econometrica 1974; 42(2): $267-272$.

20. Aseev SM. Methods of regularization in nonsmooth problems of dynamic optimization. Journal of Mathematical Sciences 1999; 94(3):1366-1393.

21. Aseev SM, Kryazhimskii AV, Tarasyev AM. First order necessary optimality conditions for a class of infinite horizon optimal control problems. Technical Report IR-01-007, International Institute for Applied Systems Analysis, Laxenburg, Austria, 2001.

22. Aseev SM, Kryazhimskii AV, Tarasyev AM. The Pontryagin maximum principle and transversality conditions for a class of optimal economic growth problems. Proceedings of the 5th IFAC Symposium on Nonlinear Control Systems, St. Petersburg, Russia, 2001; 64-68.

23. Aseev SM, Kryazhimskii AV, Tarasyev AM. The Pontryagin maximum principle and transversality conditions for an optimal control problem with infinite time interval. In Proceedings of the Steklov Institute of Mathematics, vol. 233, Moscow, 2001; 64-80.

24. Nerlove M, Arrow KJ. Optimal advertising policy under dynamic conditions. Economica 1962; 29(114):129-142. 
25. Balder EJ. An existence result for optimal economic growth problems. Journal of Mathematical Analysis and Applications 1983; 95(1):195-213.

26. Vidale ML, Wolfe HB. An operations research study of sales response to advertising. Operations Research 1957; 5(3):370-381.

27. Cesari L. Optimization: Theory and Applications. Springer: New York, 1983.

28. Filippov AF. Differential Equations with Discontinuous Righthand Sides. Kluwer Academic Publishers: Boston, 1988.

29. Dunford N, Schwartz JT. Linear Operators, Part I: General Theory. Wiley Interscience: New York, 1958.

31. Sontag ED. Mathematical Control Theory: Deterministic Finite Dimensional Systems. Springer: New York, 1998.

30. Coddington EA, Levinson N. Theory of Ordinary Differential Equations. McGraw-Hill: New York, 1955.

32 Mordukhovich B. Approximation Methods in Problems of Optimization and Control. Wiley: New York, 2001.

33. Haurie A. Existence and global asymptotic stability of optimal trajectories for a class of infinite horizon, nonconvex systems. Journal of Optimization Theory and Applications 1980; 31:515-533.

34. Mangasarian OL. Sufficient conditions for the optimal control of nonlinear systems. SIAM Journal on Control 1966; 4:139-152.

35. Khalil HK. Nonlinear Systems. Macmillan: Englewood Cliffs, NJ, 1992.

36. Kalish S. A new product adoption model with price, advertising, and uncertainty. Management Science 1985; 31(12):1569-1585.

37. Mesak HI, Clark JW. Monopolist optimum pricing and advertising policies for diffusion of new product innovations. Optimal Control Applications and Methods 1998; 19:111-136. 